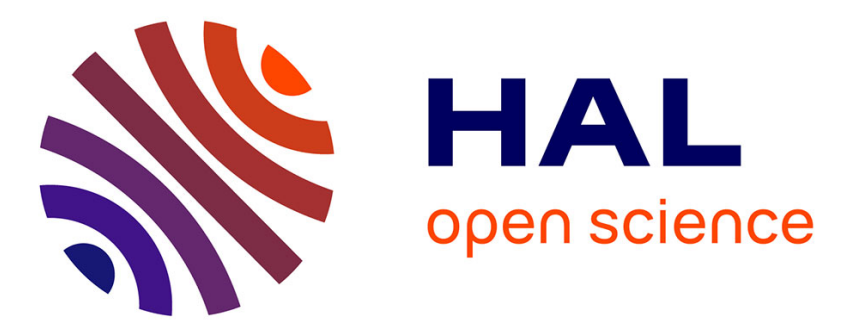

\title{
Dévoilement de données personnelles et contreparties attendues en e-commerce : une approche typologique et interculturelle
}

\author{
Caroline Lancelot Miltgen
}

\section{- To cite this version:}

Caroline Lancelot Miltgen. Dévoilement de données personnelles et contreparties attendues en ecommerce : une approche typologique et interculturelle. Systèmes d'Information et Management, 2010, 15 (4), pp.45-91. 10.3917/sim.104.0045 . hal-01117033

HAL Id: hal-01117033

https://hal-audencia.archives-ouvertes.fr/hal-01117033

Submitted on 16 Feb 2015

HAL is a multi-disciplinary open access archive for the deposit and dissemination of scientific research documents, whether they are published or not. The documents may come from teaching and research institutions in France or abroad, or from public or private research centers.
L'archive ouverte pluridisciplinaire HAL, est destinée au dépôt et à la diffusion de documents scientifiques de niveau recherche, publiés ou non, émanant des établissements d'enseignement et de recherche français ou étrangers, des laboratoires publics ou privés. 


\section{Dévoilement de données personnelles et contreparties \\ attendues en e-commerce : une approche typologique et interculturelle}

Article accepté dans la Revue SIM

\section{$\underline{\text { Auteur }}$}

\section{Caroline LANCELOT MILTGEN}

Maître de Conférences en Sciences de Gestion

GRANEM (Groupe de Recherche Angevin en Economie et Management)

Université d'Angers

UFR Droit - Economie - Gestion

caroline.miltgen@univ-angers.fr

Prix de thèse FNEGE AFM 2007 et mention spéciale du Prix de thèse « Informatique et Liberté »CNIL 2010 pour sa thèse sur les réactions des consommateurs face à la collecte de données personnelles. Responsable scientifique de deux contrats de recherche pour la Commission Européenne sur 'vie privée et systèmes d'identification électronique' (2008) et 'vie privée et management de données personnelles' (2010). Responsable d'un Master 2 en 'Management et Sécurité des Systèmes d'information de Santé' (MSSIS).

\section{$\underline{\text { Remerciements et financement }}$}

L'auteur remercie tout particulièrement les deux relecteurs anonymes, l'ensemble du comité de rédaction ainsi que le rédacteur en chef de la revue pour leur investissement, leurs remarques et leurs encouragements. L'auteur remercie également Ramon Compano, Ioannis Maghiros, Wainer Lusoli et Marguerita Bacigalupo pour la confiance qu'ils lui ont accordée lors du contrat de recherche 'European Commission - Joint Research Centre - Institute for Prospective Technological Studies Contract $\mathrm{n}^{\circ} 150876$ 2007 F1ED-FR' dont sont - en partie - issues les données utilisées pour cet article. 


\section{Dévoilement de données personnelles et contreparties attendues en e-commerce : une approche typologique et interculturelle}

\section{RÉSUMÉ}

Si les informations clients représentent une source de valeur majeure pour les entreprises, leur collecte est confrontée aux préoccupations grandissantes des consommateurs quant au respect de leur vie privée, notamment sur Internet. L'objectif de cette recherche est de proposer une typologie des internautes selon les avantages qu'ils recherchent en échange de la fourniture de données en ligne, ce qui la différencie des travaux existants qui portent plutôt sur le niveau de préoccupation. L'étude, menée sur un échantillon de 2014 répondants français et de 1258 Anglais, met en évidence quatre segments qui se retrouvent dans les deux cultures: les réticents, les désintéressés, les négociateurs et les bienveillants. La distinction des deux catégories intermédiaires, qui diffèrent par l'aspect sur lequel l'individu focalise son attention (sécuritaire vs. utilitaire), constitue une originalité théorique aussi bien qu'un atout pour les managers et responsables SI qui ne peuvent aborder la question du e-commerce qu'avec nuance et respect pour les préoccupations différentes des internautes. La comparaison entre les pays souligne des similarités en termes d'attitudes et des différences de comportements entre deux populations appartenant au même Continent mais de culture différente. Les résultats montrent notamment que les jeunes internautes anglais se sentent davantage préoccupés que les Français et qu'ils perçoivent davantage de risques à fournir des données en ligne. Au-delà d'une compréhension théorique des enjeux liés à la privacy, nos résultats confirment la nature contextuelle d'Internet et de la vie privée et offrent la possibilité d'un débat éthique autours de la question de la propriété des données à caractère personnel.

\section{Mots clés :}

Respect de la vie privée, données personnelles, typologie, avantages recherchés, Internet 


\title{
Disclosure of personal data and expected counterparties in e- commerce: a typological and intercultural approach
}

\begin{abstract}
Consumer data is a major source of value for companies. However, on the Internet, the collection of this data is increasingly difficult due to rising consumer concerns about respect for their privacy. The objective of this research is to propose a typology of Net surfers according to the advantages they seek in exchange of their personal data. This is different from previous work which mainly focuses on privacy concerns to establish cluster analysis. The results of our survey, based on 2014 French internet users and 1258 English web surfers, indicate four different groups in both countries: reticent, unconcerned, negotiator, wellintended. The two intermediate groups (unconcerned and negotiator) differ according to the way the individuals focus their attention (security verses utility). This is both theoretically original and constitutes a valuable insight for managers should they wish to apply nuance and respect for the different concerns of internet users when considering their approach to ecommerce. The comparison between the French and English samples underlines the similar attitudes and different behaviours between two populations belonging to the same Continent, with different cultures. Our findings show that young English people have more concerns about privacy than French people, resulting in greater perceived risks about data disclosure. Beyond a theoretical understanding of issues related to privacy, our results support the contextual nature of the 'Internet and privacy' and offer the possibility of an ethical debate around the issue of personal data ownership.
\end{abstract}

\section{Key words:}

Privacy, personal data, cluster analysis, benefits, Internet 


\section{INTRODUCTION}

Aujourd'hui, des données personnelles qui en disent long sur notre vie privée circulent librement dans un monde sans frontières. Les fichiers et les technologies de traçage se multiplient toujours davantage, faisant disparaître petit à petit la notion d'anonymat (Rennhard et al. 2004). Le développement des technologies de l'information et de la communication (TIC) va-t-il alors à l'encontre des libertés individuelles ? Cette traçabilité des comportements est certainement «génératrice de méfiance » (Isaac et Kalika 2001), en témoigne une sensibilité accrue de la population - et ce au niveau mondial - aux questions de vie privée (Rennhard et al. 2004) et une réticence certaine - d'une partie de cette population au moins - à dévoiler des renseignements personnels, notamment dans un cadre commercial. Pourtant, le succès de sites de réseaux sociaux de type Facebook (plus de 14 millions d'utilisateurs en France, Mars 2010 ${ }^{1}$ ) sur lequel chacun peut construire un profil parfois très détaillé, échanger des informations avec d'autres utilisateurs (y compris inconnus) et affirmer sa préférence pour une marque (devenir fan) montre que certains individus sont prêts à révéler des données personnelles voire très intimes. Ceci amène alors à se demander si tout le monde a réellement la même volonté de protéger sa vie privée, notamment en ligne. $\mathrm{Ne}$ souhaite-t-on pas plutôt aujourd'hui, comme le PDG de Facebook l'affirme ${ }^{2}$, en révéler davantage ? Et finalement, où se situe les nouvelles frontières de notre vie privée ?

Le thème du respect de la vie privée n'est certes pas nouveau. « Le chaland anonyme cède la place au client identifié puisque l'on connait désormais ses marques préférées, les quantités consommées et les modes de paiement privilégiés » remarquait déjà Lemoine en 1993. Toutefois, il revêt aujourd'hui une dimension particulière dans un contexte où, d'une part, l'exploitation de l'information client représente l'une des premières sources de valeur pour les entreprises (Ward, Bridges et Chitty 2005) et où, d'autre part, l'arrivée d'Internet est venue accroitre l'échelle avec laquelle la sollicitation et l'exploitation de données sont désormais possibles (Liu et al. 2005). Un sondage récent (Octobre 2008) réalisé pour la $\mathrm{CNIL}^{3}$ montre que les Français témoignent d'une défiance accrue à l'égard du niveau de protection de leur vie privée sur Internet, $71 \%$ le jugeant « insuffisant » et 37\% « pas du tout satisfaisant ». Certains internautes auraient ainsi renoncé à commercer en ligne (Dinev et Hart 2005/06) par crainte que leurs données personnelles soient divulguées à des tiers ou

\footnotetext{
${ }^{1}$ Statistiques Facebook au 31 mars 2010 : 14290700 utilisateurs en France

${ }^{2}$ Mark Zuckerberg, le fondateur du site Facebook, a indiqué récemment «oui, la protection de la vie privée est un concept dépassé ! A l'occasion d'une conférence, le tout jeune (25 ans) PDG de Facebook a asséné : «Les gens sont à l'aise, non seulement avec le fait de partager plus d'informations différentes, mais ils sont également plus ouverts, et à plus de personnes. La norme sociale a évolué ces dernières années. » Citations tirées du blog d'A. Bazot, PDG de l'UFC Que Choisir (http://www.alain-bazot.fr/index.php/la-vie-privee-un-concept-depasse/)

${ }^{3}$ Source : http://www.cnil.fr/la-cnil/actu-cnil/article/article//71-des-francais-jugent-la-protection-de-la-vie-privee -sur-internet-insuffisante/
} 
exploitées sans leur accord préalable. Dans le cadre d'une analyse prospective sur les libertés et l'avenir de la société informatisée, il s'avère donc nécessaire de s'interroger sur les conditions d'un échange consensuel, «point d'équilibre entre le besoin d'intimité des particuliers et le besoin de savoir des institutions » (Lemoine 2000), qu'elles soient publiques ou privées. En effet, «si la protection de la vie privée est une valeur fondamentale de la démocratie, elle doit s'imposer d'une manière ou d'une autre à l'économie de marché et à ses jeux concurrentiels. La question porte alors sur la manière d'assurer cette protection sans abîmer les ressorts de la créativité et de la compétitivité des entreprises » (Terré 2000). Or, les recherches (auteur; Hui et al. 2007), tout comme la réalité des faits, montrent que les individus sont d'accord pour fournir des données les concernant s'ils en perçoivent une contrepartie. Ce constat rejoint celui fait par Culnan et Armstrong (1999) qui indiquent que les entreprises doivent considérer la collecte de données clients comme un contrat social au cours duquel le consommateur délivre des données privées en échange de bénéfices intangibles tels qu'une plus grande qualité de service. Puisque l'écart entre les besoins des firmes et les attentes des individus semble s'accroître chaque jour, une meilleure connaissance et compréhension des stratégies permettant de promouvoir le dévoilement tout en respectant la vie privée s'avère d'une importance majeure tant d'un point de vue académique que managérial. Le dynamisme et la complexité actuelle de l'environnement numérique amplifie encore davantage cet impératif, notamment du fait de son extrême fragmentation (nouveaux internautes, variété des médias et des contenus) (Brandtzaeg 2010). Il apparaît ainsi nécessaire d'identifier la variété des comportements de dévoilement en ligne de manière plus nuancée. Si la littérature fournit déjà des typologies en matière de vie privée - essentiellement basées sur le niveau de préoccupation des individus - celles-ci ne permettent pas vraiment de décrire ni de comprendre comment les individus gèrent leurs données personnelles, en particulier dans le cadre de leurs relations avec des sites marchands. Le présent article cherche à combler ce manque en étudiant les bénéfices attendus par les consommateurs, et par là même la valeur que ces individus accordent à la fois aux données personnelles et à la protection de leur vie privée. Les travaux passés sur la vie privée portent essentiellement sur l'importance, les causes et les conséquences des préoccupations tandis que nous nous intéressons à l'un des critères majeurs de la décision de dévoilement (i.e. le type de bénéfices attendus), élargissant ainsi la vision actuelle des recherches sur ce thème. Il s'agit plus exactement d'examiner les différences - notamment, mais pas uniquement, d'ordre culturel - entre les individus à propos du dévoilement et de les classer dans des catégories pertinentes et utiles à la prise de décision. Nos résultats doivent ainsi aider les développeurs de SI à prendre en compte cette diversité et à mieux adapter leurs produits/services aux différents besoins identifiés. Ils représentent aussi 
l'opportunité pour les entreprises d'améliorer la manière et l'efficacité avec laquelle elles collectent des données, tout en s'assurant de la satisfaction des usagers. D'un point de vue académique, ils permettront d'évaluer l'hétérogénéité des comportements de dévoilement, d'en identifier les causes et de mieux comprendre les implications sociales de tels phénomènes, par exemple en matière de fracture numérique (Brandtzaeg 2010). Par ailleurs, l'étude fournira aux autorités publiques un aperçu des attitudes des citoyens à cet égard, rendant possible le développement de politiques visant à «maximiser les bénéfices sociaux des médias numériques » (Europa 2009) tout en prenant davantage en compte la diversité des avantages «économiques » recherchés. Enfin, l'étude devrait permettre d'élargir le spectre d'analyse des recherches existantes, d'un point de vue historique et géographique (i.e. culturel). Jusqu'ici, la majorité des travaux a en effet été menée avant 2005 et concerne des échantillons de consommateurs ou d'internautes nord-américains. Or, l'évolution extrêmement rapide des pratiques doit amener à réactualiser régulièrement ces travaux. De plus, l'environnement technologique et l'encadrement juridique diffèrent notablement d'un continent et d'un pays à l'autre (Dinev et al. 2006), amenant dès lors à s'interroger sur les comportements de dévoilement au niveau européen et, en particulier, au niveau français. Par ailleurs, puisqu'il existe des différences de préoccupation selon les cultures (Dinev et al. 2006, Milberg et al. 1995), il parait intéressant d'étudier si ces différences persistent dans deux pays appartenant au même continent (l'Europe) mais culturellement différents (France et Angleterre), pour compléter les travaux actuels. Dans l'économie globalisée qui est la nôtre, ces recherches multiculturelles s'avèrent essentielles pour adapter les modèles et les pratiques aux écarts identifiés et ainsi assurer des échanges fructueux et satisfaisants pour tous. Du fait des problèmes de sécurité et de confidentialité, ceci est d'autant plus indispensable en ce qui concerne le partage de données.

L'objectif majeur de cette contribution est donc d'étudier les comportements actuels de dévoilement de données en ligne avec, pour but, de dresser une Typologie des internautes sur la base des Avantages Recherchés en Echange du Dévoilement (TARED). Nous cherchons à savoir s'il existe des types d'internautes plus ou moins enclins à se dévoiler et quel est leur profil. La principale originalité de ce travail est de s'intéresser aux contreparties attendues par les internautes en échange de la communication de données personnelles alors que les travaux existants étudient davantage le niveau de préoccupation. L'analyse typologique est menée sur des internautes français puis reconduite sur des internautes anglais, à des fins de validation et de comparaison. Dans une optique prescriptive, nous testons aussi des variables, autres que culturelles, susceptibles de caractériser le profil des individus appartenant aux types identifiés et offrant ainsi des clés d'actions aux managers de SI. 


\section{FONDEMENTS THEORIQUES}

Notre cadre théorique repose sur les concepts de vie privée et de propriété des données personnelles afin d'identifier, d'une part, les types d'atteintes à l'intimité des individus (tableau 1) et, d'autre part, les conditions nécessaires au dévoilement d'informations. Emerge alors l'idée d'échange ou de contrat social (Culnan et Armstrong 1999) entre l'entreprise qui collecte les données et le client qui accepte de les fournir en contrepartie de bénéfices économiques ou sociaux. Le fait que les individus ne soient pas tous sensibles au même type d'avantages justifie l'intérêt d'un cadre taxonomique des profils de consommateurs.

\subsection{Le concept de vie privée}

La délimitation de ce qui constitue la vie privée (privacy) est difficile et délicate parce qu'il s'agit d'un sujet complexe (Dinev et al. 2006) et multidimensionnel. Il est en effet question d'un phénomène individuel et psychologique (Woodman et al. 1982) ayant des composantes à la fois cognitives (croyances relatives, notamment, au type de données considérées comme privées) et affectives (sentiments et émotions liées aux pratiques de sollicitation et de dévoilement). De plus, le désir d'intimité est fortement dynamique : les gens s'engagent en effet dans un processus d'ajustement perpétuel au cours duquel le désir de vie privée est comparé au désir de communication avec les autres (Westin 1967). Cet ajustement a lieu dans un contexte de forces variées telles que la pression des autres ou les normes sociales, qui varient d'un moment et d'une situation à l'autre (Kimmel 1996).

Globalement, les réponses les plus courantes à la définition de la vie privée tombent dans trois catégories principales (Tableau 1) : 1) le droit d'être laissé seul (ou « right to be let alone »), 2) le contrôle de l'accès aux données et 3) la liberté face au contrôle exercé par autrui. Celles-ci représentent autant de préoccupations possibles pour un individu et caractérisent le type de menace concernée, respectivement : physique (atteinte au droit à être laissé seul), informationnelle (menace sur le droit de contrôler l'accès aux données), psychologique et/ou relationnelle (menace sur le droit de contrôler sa vie privée). Le point commun à toutes ces définitions de la vie privée est la notion de contrôle. La majorité des auteurs se focalisent en effet sur ce droit à contrôler l'accès aux données et opérationnalisent la vie privée (privacy) comme la préoccupation liée à une possible perte de contrôle sur les informations personnelles dévoilées (informational privacy concern) (e.g. Dinev et al. 2006). Le droit au respect de la vie privée peut ainsi se définir comme : «le droit de protéger ses données personnelles et de contrôler la collecte et l'utilisation future de celles-ci ». Le débat se concentre dès lors sur l'opposition entre le droit du client de décider ce qui sera fait des données qu'il accepte de fournir et celui de l'entreprise de les utiliser dans un but commercial. 
Tableau 1 - Les différentes conceptualisations de la vie privée

Conceptualisation

Description

«Droit d'être laissé seul $»($ ou « right to be let alone »)

Possibilité de s'isoler, de placer une barrière physique entre soi et l'extérieur qui fait ainsi écho aux notions d'espace personnel et de territorialité. Cet aspect physique et/ou spatial de la protection est luimême axé sur l'idée de séparation entre les sphères publique et privée.

Droit de déterminer dans quelle mesure des informations nous concernant

«Contrôle de

l'accès à ses

informations

personnelles » peuvent être révélées aux autres. Selon cette perspective, les individus sont protégés quand ils sont capables de contrôler les impressions suscitées par les données qu'ils fournissent. Cela s'opère par le biais d'une procédure de divulgation sélective d'informations et par la régulation de la dissémination de ces données.

Liberté et protection de son individualité face aux pressions d'autrui. La

«Liberté face au contrôle des notion inclut un aspect psychologique lié à la pression pouvant être exercée par autrui pour déterminer nos pensées et nos attitudes. A travers ce type de protection, l'individu réduit donc le niveau de pression susceptible d'être exercé par autrui ce qui lui permet d'améliorer son autonomie et de réduire sa vulnérabilité.

\subsection{La propriété des données personnelles et les conditions du transfert}

Même si de nombreux chercheurs - y compris aux USA - considèrent la vie privée comme étant intrinsèquement liée à la dignité humaine (Whitley 2009), force est de constater que la vie contemporaine conduit souvent à une perte d'intimité puisqu'elle requiert de fournir toujours davantage de données (Hough 2009). La question centrale concerne alors les droits de propriété associés à ces informations. Or, si l'objectif de la législation, notamment au niveau européen, est « d'assurer les conditions de maîtrise des personnes sur ces données, en leur reconnaissant un véritable droit à déterminer l'usage qui doit être fait des données personnelles » (Belen 2005), cette volonté se heurte au fait qu'Internet représente un «nouvel environnement pour les comportements non éthiques » (Freestone et Mitchell 2004, Roman 2006), notamment en ce qui concerne la collecte de données, l'envoi d'emails non sollicités et la sécurité des transactions (Roman et Cuestas 2008). Toutefois, puisque les données clients constituent désormais un élément clé de la valeur des entreprises, il s'avère de plus en plus indispensable pour elles de tenir compte des préoccupations des clients à ce sujet, notamment en obtenant les données de manière légitime et en ne les utilisant qu'avec le consentement préalable de leur propriétaire. Mascarenhas et al. (2003) proposent alors de considérer les droits associés aux données comme des «privilèges» que les clients accepteraient de transférer aux firmes sous réserve de conditions satisfaisant ce partage. Les sites pourraient alors motiver et compenser de tels transferts par le biais de bénéfices correspondant aux avantages recherchés par leurs clients (e.g. baisse de prix, information privilégiée, confort). 
La littérature (e.g. Culnan et Armstrong 1999) confirme que les consommateurs sont prêts à s'engager dans cette démarche si les bénéfices perçus à le faire excèdent les risques (dont ceux liés au respect de la vie privée). Elle indique aussi que tous les individus ne sont pas égaux à cet égard. Les analyses typologiques semblent dès lors trouver tout leur sens.

\subsection{Intérêt et caractéristiques des typologies}

Les typologies d'utilisateurs-consommateurs correspondent à une catégorisation d'individus en différentes classes qui décrivent les préférences et les usages de ces derniers sur la base de critères d'analyse tels que, dans le cas qui nous intéresse ici, les avantages recherchés en échange de la fourniture de données personnelles. D'un point de vue académique, la classification - et la description des catégories qui en découle - jouent un rôle fondamental dans le développement d'une discipline (Hunt 1983) et sont utilisées depuis longtemps dans certains domaines, notamment en psychologie à travers l'étude des traits de personnalité (John \& Srivastava 1999). Elles connaissent par ailleurs un regain d'intérêt actuellement, en témoigne une méta-analyse sur les typologies d'usage des médias qui dénombre 10 études (sur 22) publiées après 2006 (Brandtzaeg 2010). En sciences sociales, les typologies sont utilisées pour classer les comportements complexes en «modèles caractéristiques » permettant alors d'explorer la nature et les conséquences des catégories ainsi identifiées (Johnson \& Kulpa 2007). De telles catégories constituent la base des techniques de segmentation dont l'importance est reconnue, notamment pour l'adaptation des produits/services (LaPlaca 1997) et une meilleure allocation des ressources (Freytag et Clarke 2001). Malgré des limites avérées (pluralité des méthodes et des critères, validité des résultats du fait des données mensongères, persistance des catégories dans l'espace et dans le temps), l'utilisation de telles techniques est courante aussi bien en marketing (e.g. Wedel et Kamakura 2000) qu'en SI (e.g. Burnett 2000). Elles offrent en effet des clés extrêmement utiles pour comprendre les comportements des individus - et leur évolution - et permettent d'envisager des actions de personnalisation pertinentes dans le cadre de la gestion de la relation client.

Cette technique a notamment montré son intérêt dans l'étude des usages des médias tels qu'Internet (Brandtzaeg 2010) où, au-delà de la différenciation binaire utilisateur/nonutilisateur, il existe de nombreuses variations d'usages (Zillien \& Hargittai 2009) qu'il est nécessaire d'identifier, d'une part, pour développer des solutions mieux adaptées à la population et, d'autre part, pour mieux comprendre les implications théoriques et sociales d'un tel phénomène. Ceci est également valable pour le sujet qui nous intéresse ici à savoir l'acceptation des clients de fournir des données les concernant et les conditions d'un tel consentement (respect de la vie privée, bénéfices obtenus en échange, ...). 


\subsection{Les typologies de préoccupation de respect de la vie privée}

Les individus diffèrent sensiblement quant à leur niveau de préoccupation pour le Respect de la Vie Privée (RVP) d'une part et leur acceptation de dévoiler des données personnelles d'autre part ${ }^{4}$. Des recherches menées auprès de consommateurs ou d'internautes, majoritairement Etasuniens, permettent de distinguer 3 ou 4 groupes d'individus (Annexe 1).

La première typologie réalisée dans ce domaine (Westin 1967) met en évidence trois groupes d'individus - Etasuniens - ayant des attitudes contrastées face aux pratiques de collecte et d'utilisation de leurs informations personnelles hors ligne. Le premier groupe (les “fondamentalistes", $25 \%$ des consommateurs interrogés), est constitué de personnes estimant que les citoyens ne contrôlent pas suffisamment leurs données personnelles et que la majorité des entreprises utilisent ces informations de manière abusive. Les individus du second groupe (“non préoccupés", 20\%) font, dans l'ensemble, confiance aux entreprises qui leur demandent des informations personnelles. Entre ces deux extrêmes, "les pragmatiques" (55\%) recherchent avant tout leur propre intérêt. Ils analysent les bénéfices proposés en échange ainsi que les risques encourus et les garanties offertes et se décident en conséquence. Une telle typologie souligne l'importance de cette catégorie intermédiaire qui constitue le cœur de cible de nombreuses entreprises. Les " pragmatiques" semblent en effet être les plus faciles à convaincre, les autres se décidant davantage sur la base de convictions personnelles.

D'autres travaux menés sur des échantillons d'internautes aboutiront, quant à eux, à une classification en quatre groupes. A côté des «fondamentalistes » et «peu préoccupés », on trouve ainsi, d'une part (Spiekerman et al. 2001), les “identity concerned" (20\%), préoccupés de devoir fournir des données d'identification (nom et email) et les " profile concerned" (26\%), concernés par les questions sociodémographiques ou portant sur les centres d'intérêt ; et, d'autre part (Sheehan 2002), les "circonspects" (38\%) et "prudents" $(43 \%)$, respectivement assez et très préoccupés par ce qui porte atteinte à leur vie privée.

Sur l'ensemble des études typologiques menées jusqu'à présent sur ce thème, une seule (Spiekerman et al. 2001) a été réalisée sur un échantillon européen (en l'occurrence allemand) et une autre (Hann et al. 2002) a été conduite dans deux pays différents (en l'occurrence Etats-Unis et Singapour). Dans les deux cas, si le nombre de groupes identifiés

\footnotetext{
${ }^{4}$ Bien que conceptuellement délicate, la distinction entre préoccupation pour le respect de la vie privée et acceptation de fournir des données personnelles, n'en est pas moins importante. D'une part, l'acceptation (ou le refus) de fournir des données n'est qu'un moyen parmi d'autres de contrôler l'accès à ses données, ce contrôle représentant un élément de la vie privée auquel doivent s'ajouter deux autres dimensions (cf. tableau 1). Par ailleurs, si la littérature montre effectivement que ces deux construits sont conceptuellement distincts (e.g Dinev and Hart 2006), la démonstration empirique d'une relation causale entre eux est plus délicate. En effet, si la majorité des recherches confirment le rôle antécédent de la préoccupation (e.g. Farag and Krishnan 2003; Malhotra et al. 2004; Van Slyke et al. 2006), d'autres concluent sur l'absence possible d'un tel lien ou sur une relation plus ambiguë entre les deux (auteur 2005).
} 
dans l'échantillon non-américain (allemand ou de Singapour) est identique à celui trouvé dans l'échantillon Etasunien (3 ou 4 groupes), les caractéristiques des individus appartenant à ces groupes diffèrent. Ainsi, les «vendeurs de données» Etasuniens ont une plus grande expérience de dévoilement que les «vendeurs de données » de Singapour (Hann et al. 2002).

La convergence des résultats issus de ces travaux confirme, d'une part, les différences au sein de la population en ce qui concerne le niveau de préoccupation pour le RVP et, d'autre part, une répartition en trois ou quatre groupes. Dans notre cas, plutôt que d'étudier l'attitude générale face au RVP, comme l'ont fait un grand nombre d'auteurs jusqu'ici, nous avons préféré travailler sur les bénéfices recherchés par les individus en échange de leurs données. Ce type de variable, bien que différent des questions d'ordre général posées par Westin (1996), offre l'avantage de correspondre à l'esprit d'origine de ce type de typologie et peut s'avérer utile à la définition des catégories ainsi trouvées : les «pragmatiques » attendraient un bénéfice en échange de leurs données, tandis que les «non préoccupés » refuseraient de se dévoiler, quel que soit le type de bénéfice proposé. De plus, ce choix répond aussi à un principe de base établi au sein de la littérature, selon lequel le comportement de dévoilement dépend - notamment et principalement - des bénéfices proposés par l'entreprise qui sollicite les données. Les recherches expliquent en effet que les individus réalisent un «privacy calculus » (Laufer et Wolfe 1977) c'est-à-dire qu'ils évaluent les coûts et bénéfices de la divulgation, avant de décider de transmettre ou non des informations. Des études montrent ainsi que certains individus peuvent accepter de fournir des données en échange de cadeaux ou de catalogues (Schwartz 2000). Ce principe de «compensation" est conforme aux théories de l'échange social et des relations interpersonnelles (Taylor et alii 1969), qui sont souvent utilisées dans les recherches sur le RVP. Enfin, de nombreux travaux confortent l'hypothèse selon laquelle une partie, au moins, des individus évalue les bénéfices avant de décider de divulguer ou non leurs données (Castaneda et Montoro 2007 ; Chellappa et Shivendu 2007 ; Hui et al. 2007). Sur cette base, nous formulons une première hypothèse :

H1 : Il existe, au sein de la population étudiée, des différences en ce qui concerne le type d'avantages recherchés en échange du dévoilement de données personnelles

Puisque l'aspect culturel exerce une influence non négligeable sur la préoccupation des individus pour le RVP (Milberg et al. 1995), nous nous efforcerons de compléter les travaux passés en travaillant sur une population d'internautes français et en comparant nos résultats à des internautes provenant d'un autre pays européen (en l'occurrence l'Angleterre). Ceci permettra d'identifier l'effet culturel dû à la nationalité de l'individu tout en contrôlant l'effet 
lié au Continent (auquel la même réglementation en matière de protection des données personnelles s'applique), ce qui ne s'est jamais fait dans les études passées. En référence aux études multiculturelles déjà menées sur ce sujet en SI - notamment à Dinev et al. (2006) nous avons choisi deux pays culturellement différents (France et Angleterre), que l'on se réfère aux cinq dimensions de Hofstede (2001) ou à la catégorisation «forte confiance » (Angleterre) vs. «faible confiance» (France) de Fukuyama (1995). Puisque les pays méditerranéens (Italie, France) auraient un niveau de préoccupation pour le RVP plus faible que celui observé dans les pays à la culture individualiste (USA, pays anglo-saxons), nous pensons que cela aura également une incidence sur le type d'avantages recherchés :

H2 : Le type d'avantages recherchés en échange du dévoilement de données diffère selon la culture (anglo-saxonne vs. française) de la population étudiée

Par ailleurs, nous cherchons à identifier des variables de profil (attitudinales, d'expérience, comportementales ou sociodémographiques) permettant de discriminer la prédisposition au dévoilement de données eu égard aux types d'avantages recherchés en échange. Tout d'abord, puisque l'attitude face au dévoilement, souvent mesurée par la préoccupation pour le RVP, discrimine la population (Annexe 1), nous pensons que d'autres variables d'attitude face au RVP sont également susceptibles d'influencer le type d'avantages recherchés en échange :

H3: Le type d'avantages recherchés en échange du dévoilement de données personnelles diffère selon l'attitude de la population eu égard aux questions de RVP

D'autres travaux laissent penser que le comportement habituel de dévoilement aurait aussi un effet discriminant (Hann et al. 2002). Les individus les plus enclins à délivrer des informations auraient ainsi une plus grande expérience de partage de données que les autres. De plus, ils accepteraient plus facilement de s'enregistrer sur un site et fourniraient moins fréquemment des données incomplètes (Sheehan 2002). A l'opposé, les individus peu habitués à se dévoiler auraient tendance à fournir des données erronées. D'où l'hypothèse :

H4: Le type d'avantages recherchés en échange du dévoilement de données personnelles diffère selon les comportements habituels de partage

Des auteurs se sont aussi interrogés sur l'impact de facteurs d'expérience tels que l'expérience d'Internet ou l'expérience passée en matière d'achat en ligne. Plusieurs travaux soulignent ainsi que l'expérience en ligne serait peu ou pas discriminante, que ce soit en matière d'utilisation d'Internet (i.e. : surf, lecture des emails) (Sheehan 2002) ou d'expérience d'achat (Jensen, Potts et Jensen 2005). Nous en déduisons l'hypothèse suivante :

H5: Le type d'avantages recherchés en échange du dévoilement de données personnelles diffère selon l'expérience (en ligne) de la population étudiée 
Concernant les recherches portant sur les différences d'ordre sociodémographique, malgré des résultats controversés - certains auteurs soulignant l'absence de pouvoir discriminant de ce type de variables (Cranor, Reagle et Ackerman 1999) - plusieurs travaux sur la préoccupation pour le RVP indiquent des différences intergroupes significatives selon l'âge (Sheehan 2002), le niveau d'éducation (Sheehan 2002) et le genre (Westin 1996) des individus. Ceci nous amène à supposer que le type d'avantages recherchés pourrait également varier selon les caractéristiques démographiques des individus, comme formulé dans l'hypothèse suivante :

H6: Le type d'avantages recherchés en échange du dévoilement de données personnelles diffère selon les caractéristiques sociodémographiques de la population

En résumé, pour d'une part différencier et caractériser les individus appartenant à chaque classe et, d'autre part prendre en compte un maximum de facteurs ${ }^{5}$ susceptibles d'intervenir dans la décision de dévoilement de soi, nous intégrons à la fois des variables liées au macroenvironnement, comme la culture (qui sera évaluée à travers la nationalité de l'individu française vs. anglaise), et des facteurs liés au microenvironnement donc à l'individu lui-même tels que les variables attitudinales (dont le niveau de protection perçu, la préoccupation pour le RVP et la confiance dans le média Internet), d'expérience (ancienneté et type de compétences sur Internet) et comportementales (mesures de protection adoptées notamment) ainsi que les facteurs sociodémographiques. Le choix des variables au sein de chaque facteur du microenvironnement est lié, d'une part, à une analyse approfondie de la littérature et des recherches existantes sur le RVP et, d'autre part, aux résultats d'une étude qualitative qui sera présentée ultérieurement. Par ailleurs, loin de considérer le dévoilement de données personnelles comme une tendance systématiquement stable de l'individu, nous introduisons aussi dans notre analyse des éléments permettant d'étudier la variabilité de l'échange en fonction de facteurs situationnels tels que le type d'interlocuteur sollicitant les données (à travers la confiance envers celui-ci) et le type de données demandées par le site. Ce type de facteur contextuel a précédemment été identifié comme influençant la décision de dévoilement (auteur) et nous pensons que cette influence serait potentiellement liée à son impact sur le type d'avantages recherchés à travers le dévoilement, d'où l'hypothèse :

H7: Le type d'avantages recherchés en échange du dévoilement de données personnelles diffère selon le contexte dans lequel la population est sollicitée

\footnotetext{
${ }^{5} \mathrm{Si}$ nous sommes conscients, vu la complexité du phénomène, de ne pas pouvoir prendre en compte la totalité des facteurs susceptibles d'intervenir dans une telle décision, nous nous attachons néanmoins à travers cette différenciation des comportements à introduire le maximum d'éléments possible afin de réduire au maximum cette limite. Ainsi, les effets liés à l'histoire sont traduits à la fois par l'aspect culturel (macro-économique) et par l'expérience de l'individu (micro-économique). De même, les éléments cognitifs sont - au moins en partie repris à travers les variables d'expérience et les variables attitudinales.
} 
Le schéma 1 présente le cadre conceptuel retenu pour cette analyse typologique des avantages recherchés en échange du dévoilement de données personnelles sur un site marchand (Typologie dénommée de l'acronyme TARED). Ce schéma rend bien compte de la diversité des éléments pris en compte dans cette recherche avec six catégories de variables différentes.

Schéma 1 - Modèle conceptuel en vue d'une typologie des avantages recherchés en échange du dévoilement (TARED)

Variables descriptives $\quad$ Variable typologique

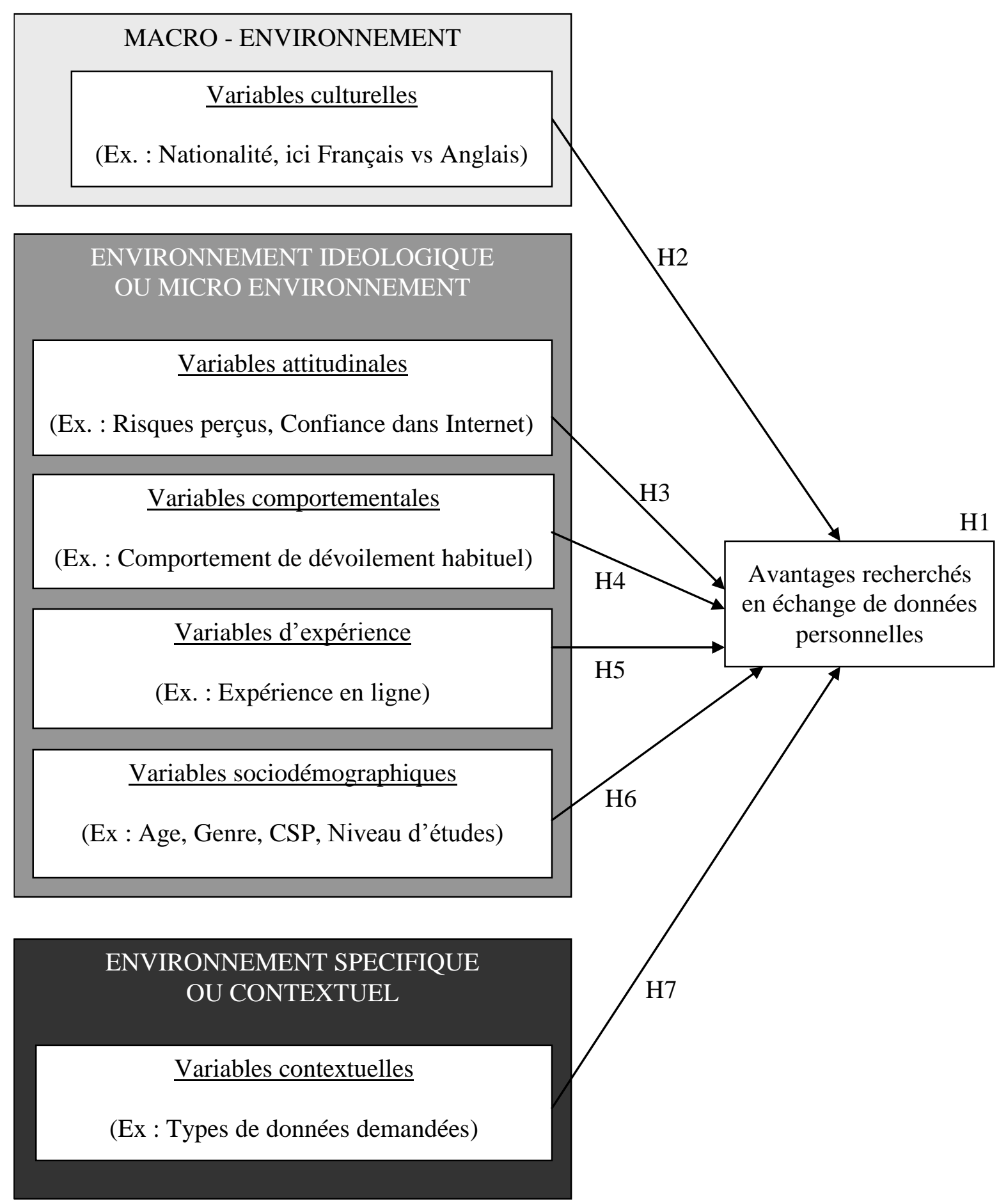




\section{METHODOLOGIE}

Pour atteindre nos objectifs, nous avons d'abord mené une étude qualitative dans le but d'identifier les avantages recherchés par les internautes en échange de la fourniture de données personnelles et le type de variables susceptibles de les influencer. Nous avons ensuite administré un questionnaire visant à déterminer si des types d'internautes correspondant à différentes combinaisons d'avantages recherchés se formaient. Le questionnaire contenait aussi l'ensemble des variables de profil identifiées comme potentiellement influentes dans le but de déceler le profil moyen des individus appartenant à chacun de ces groupes. Nous présentons ci-après l'ensemble de la démarche suivie.

\subsection{L'étude qualitative exploratoire}

Avant de bâtir la typologie, une étude qualitative exploratoire a été menée. Le but était notamment de se familiariser avec les construits étudiés (respect de la vie privée, données personnelles, avantages recherchés). Des entretiens individuels semi-directifs d'une durée moyenne d'1h30 ont été réalisés auprès de 9 internautes français aux profils contrastés en termes sociodémographiques et d'usages d'Internet. Durant ces entretiens, les participants étaient invités à définir ces concepts avec leurs propres mots. Il leur était aussi demandé de décrire leur comportement de dévoilement vis-à-vis de sites commerciaux et d'expliquer les critères utilisés pour décider de fournir ou non les données personnelles demandées. Enfin, nous cherchions à identifier les besoins et motivations en termes de protection de la vie privée. Les résultats (auteur 2003) confirment que la valeur perçue de l'échange (rapport coûts/bénéfices de la divulgation) - et donc le type d'avantages susceptibles d'être reçus en échange - est un élément déterminant dans la décision de dévoilement de données personnelles sur Internet. Plusieurs répondants reconnaissent en effet l'idée d'échange avec la firme qui les sollicite, expliquant accepter de divulguer des données s'ils ont quelque chose en retour («je l'envisage comme la contrepartie quand il y a des services gratuits. C'est plus un échange de bons procédés que vraiment un bénéfice que j'en retire de manière directe et personnelle »). Par ailleurs, les internautes distinguent clairement deux grandes catégories d'avantages pouvant être obtenus : des bénéfices hédonistes (« ce genre de trucs, ça m'amuse de remplir les petites cases ») d'une part, et utilitaires («je coche cadre dans l'optique où ils vont m'envoyer des offres qui vont correspondre à mes goûts, aux goûts des cadres ») d'autre part. On note ainsi une opposition entre ceux qui concèdent le côté intéressé de leur démarche et ceux qui expliquent donner souvent des renseignements gratuitement, par envie de «faire une bonne action». Par ailleurs, parmi les critères susceptibles d'influencer le dévoilement et le type d'avantages recherchés, nous identifions effectivement: les variables attitudinales (confiance, risques perçus et préoccupation pour le 
RVP notamment), les variables d'expérience en ligne (ancienneté et type d'usage), les comportements habituels en termes de dévoilement et de protection de la vie privée et les caractéristiques sociodémographiques classiques (âge, genre, CSP, niveau d'études).

\subsection{Etude quantitative : instrument de mesure et mode de collecte}

L'objectif de l'étude quantitative est d'appréhender l'attitude des internautes en matière de protection de la vie privée et d'évaluer les pratiques de transmission de données personnelles en ligne, notamment à travers le type d'avantages recherchés en échange. Sur la base de la littérature et des résultats de la pré-étude exploratoire, dix items reflétant les différents types d'avantages recherchés en échange de la fourniture de données personnelles étaient proposés aux répondants et ont été utilisés pour réaliser la typologie. Outre ces dix items, l'instrument de mesure développé (Annexe 2) ${ }^{6}$ comprend aussi de nombreuses variables descriptives visant à comparer les groupes ainsi obtenus. Après avoir été pré-testé auprès d'un échantillon de 117 répondants, le questionnaire a été administré en ligne. Après avoir cliqué sur le lien proposant de participer à une enquête sur leurs pratiques d'Internet, les participants étaient conduits sur la page d'accueil du questionnaire qui rappelait les principes et l'objectif de l'étude avant d'inviter à répondre aux questions.

\subsection{Choix et description de l'échantillon}

L'enquête a été administrée en ligne auprès de jeunes (15-25 ans) internautes en France et en Angleterre. Nous avons volontairement choisi ce pays culturellement éloigné pour enrichir au maximum la comparaison avec l'échantillon français, d'une part, et avec les travaux passés qui concernent majoritairement la population anglo-saxonne, d'autre part.

Les 15-25 ans ont grandi avec les nouvelles technologies ${ }^{7}$ et ont donc très massivement adopté le média Internet. Ainsi, 88\% des jeunes Européens âgés de 16-24 ans utilisent Internet contre $60 \%$ pour l'ensemble de la population de l'EU27 (Eurostat, 2009). Ils y ont très largement recours pour de nombreuses activités quotidiennes (chat et travail scolaire notamment). Les pratiques des jeunes sur la toile sont également souvent en avance comparées à celles des internautes moyens, ce qui fait d'eux de possible leaders d'opinion en matière de TIC. Par ailleurs, les internautes sont généralement plus jeunes et plus éduqués que le consommateur moyen (Mc Knight et al. 2002), ce qui rapproche notre échantillon de la population mère : les utilisateurs d'Internet. Nos répondants reflètent donc assurément les internautes d'aujourd'hui (Lueg et al. 2006) voire de demain (Lusoli et Miltgen 2009) dans la

\footnotetext{
${ }^{6}$ Pour rappel, l'instrument de mesure développé provient à la fois d'une revue de la littérature et d'une pré-étude qualitative auprès d'internautes français. Les items sont donc en partie issus des verbatims des répondants.

${ }^{7}$ Les anglo-saxons les appellent les "digital natives" ou "Generation Y".
} 
mesure où : 1) ce sont les futurs citoyens adultes ; 2) ils sont très technophiles; 3) ils tendent à adopter très rapidement les nouveautés proposées sur la toile (comme ce fut le cas avec les réseaux sociaux) et 4) ils géreront bientôt l'économie numérique (Tweney 1998). Enfin, les jeunes de 15-25 ans représentent 11 à 16\% de la population européenne selon le pays, ce qui constitue une part non négligeable de la population. Pour toutes ces raisons, il nous paraissait important de connaitre leur avis et leurs pratiques en matière de dévoilement de données.

L'enquête a été menée en partenariat avec la société Millemercis en utilisant leur base de données Elisa ${ }^{8}$. Pour augmenter la validité externe de l'étude, l'échantillon (liste des emails à solliciter) a été sélectionné dans cette base de données Elisa sur la base de quotas (sur les critères d'âge et de sexe), de manière à garantir sa représentativité par rapport à la population de base (jeunes internautes français et anglais). Le questionnaire a ainsi été envoyé par email à 128000 internautes français et 140000 Anglais dont l'adresse email avait été sélectionnée. Au final, 2014 participants français et 1258 participants anglais ont répondu à l'intégralité du questionnaire'. Le tableau 2 résume les caractéristiques de l'échantillon dans chaque pays.

Tableau 2 - Description de l'échantillon

\begin{tabular}{llcc}
\hline Variables descriptives & Niveaux & $\begin{array}{c}\text { France } \\
\mathrm{N}=2014\end{array}$ & $\begin{array}{c}\text { Angleterre } \\
\mathrm{N}=1258\end{array}$ \\
\hline \multirow{2}{*}{ Genre } & Masculin & $39.8 \%$ & $64.8 \%$ \\
\multirow{3}{*}{ Lieu d'habitat } & Féminin & $60.2 \%$ & $35.2 \%$ \\
& Métropole & $20.1 \%$ & $31.7 \%$ \\
\multirow{2}{*}{ Niveau d'études } & Zone urbaine & $50.6 \%$ & $38.5 \%$ \\
& Zone rurale & $29.3 \%$ & $29.8 \%$ \\
& < ou = Baccalauréat & $39.9 \%$ & $54.8 \%$ \\
Catégorie Socioprofessionnelle & Deug-Licence & $40.3 \%$ & $30.9 \%$ \\
& Master ou Doctorat & $18.5 \%$ & $14.2 \%$ \\
& Scolaires/Etudiants & $55.5 \%$ & $28.5 \%$ \\
Ancienneté sur Internet & Employés & $24.2 \%$ & $13.9 \%$ \\
& Sans emploi & $9.0 \%$ & $5.6 \%$ \\
& Autres & $11.4 \%$ & $51.9 \%$ \\
& Inférieure à 5 ans & $35.2 \%$ & $25.0 \%$ \\
Fréquence de connexion à Internet & Supérieure à 5 ans & $64.8 \%$ & $75.0 \%$ \\
& Plusieurs fois/jour & $86.4 \%$ & $79.3 \%$ \\
& Plusieurs fois/semaine & $9.7 \%$ & $14.9 \%$ \\
& Moins souvent & $3.8 \%$ & $4.7 \%$ \\
& & $0.1 \%$ & $1.1 \%$ \\
\hline
\end{tabular}

\footnotetext{
${ }^{8}$ Elisa est un programme opt-in permettant d'envoyer des offres promotionnelles par courrier, email et SMS. Les partenaires de Millemercis collectent des données comportementales sur leurs clients qui lui sont ensuite confiées pour l'hébergement, le nettoyage, la gestion et la location. La base de données contient environ 9 millions d'individus en Europe. Elle est donc multiculturelle et offre l'avantage d'être à la fois très réactive et de permettre une sélection d'échantillons représentatifs de la population de base.

${ }^{9}$ La réactivité à l'email a été nettement supérieure en France, tant du point de vue du taux d'ouverture $(37 \%$ vs. $14 \%$ en Angleterre) que du taux de clic (7,1\% vs. 2,1\%) alors que c'est l'inverse pour le taux de réponse (49\% vs. 87\%). Notons que 4485 questionnaires français et 2631 questionnaires anglais ont été obtenus au final mais que nous avons choisi de sélectionner uniquement les questionnaires complets.
} 
Si l'on compare le profil de nos répondants à celui du jeune internaute français et/ou anglais (Annexe 3), notre échantillon apparaît plus féminin que la moyenne en France et plus masculin sur l'échantillon anglais. Les caractéristiques sont par contre très proches en ce qui concerne la CSP, notre échantillon comprenant notamment une proportion d'étudiants similaire à celle trouvée dans les populations française et anglaise ${ }^{10}$. Notre échantillon n'est donc pas entièrement représentatif de la population des internautes français et anglais puisqu'il est composé de jeunes utilisateurs, plutôt de sexe féminin (Français) ou masculin (Anglais). Il s'avère toutefois assez représentatif de la population des jeunes internautes dans ces deux pays, en termes d'usages. Ainsi, du point de vue de l'utilisation d'Internet, les profils de nos répondants sont proches de ceux de la population du même âge en ce qui concerne les activités réalisées (emails, blogs, chats). Notre échantillon comprend des individus aux pratiques matures, en atteste la fréquence de connexion particulièrement élevée $(86,4 \%$ des individus se connectent plusieurs fois par jour dans l'échantillon français). Leur âge est tel qu'ils préfigurent les internautes de demain. Il s'agit donc de personnes dont il est important de connaître l'opinion en matière de dévoilement, afin d'évaluer l'impact de ce phénomène sur la population Internet dans son ensemble, et sur les internautes du futur, en particulier.

\subsection{Objectif et démarche de l'analyse typologique}

Notre objectif est d'établir une typologie des internautes selon les avantages recherchés en échange de la divulgation de leurs données personnelles sur Internet (TARED). Plus particulièrement, nous cherchons à connaître les motivations au dévoilement sur un site marchand et à comprendre comment les individus se comportent. Nous voulons aussi identifier d'éventuelles similarités - ou au contraire différences - culturelles afin de vérifier si, comme le prédit S. Campbell (2007), « les différences nationales disparaissent à long terme ».

L'étude est menée sur dix types d'avantages (ou contreparties) possibles : gain de temps, meilleur service, informations pertinentes, rester en contact (réseau), baisse de prix, plaisir, offres personnalisées, cadeaux, sécurité, rendre service ou bonne action $(\mathrm{BA})^{11}$. Ces 10 items offrent l'avantage de répondre aux deux contraintes à respecter dans le choix des variables à inclure dans l'analyse typologique (Thiétart 2003): contraintes de suffisance (n'omettre aucune variable pertinente) ${ }^{12}$ et de non redondance.

\footnotetext{
${ }^{10}$ Il est normal de trouver une forte propension de scolaires et d'étudiants dans une population de 15-25 ans.

${ }^{11}$ Ici l'avantage recherché et la motivation qui en découle sont de nature altruiste. Il s'agit du sentiment de faire une bonne action, de rendre service à la communauté. Cet avantage recherché est surtout (mais pas uniquement) présent dans le cadre de la participation à des enquêtes, notamment si elles sont d'utilité publique. D'après les résultats de l'étude qualitative, cette motivation est assez présente chez certains individus et peu chez d'autres.

${ }^{12}$ Le respect de ce critère s'avère contraignant puisqu'il est lié à l'étude de la littérature (pour couvrir l'ensemble du domaine) et à l'étude qualitative exploratoire (bénéfices attendus indiqués oralement par les participants). Notons que cette phase a également permis de s'aider des verbatims des répondants pour formuler les items.
} 
Une première analyse (SPSS 17.0) sur ces 10 items relatifs à la variable 'avantages recherchés' est conduite sur l'échantillon français (2014 observations), et ce, en 3 temps :

(1)Comme conseillé par Ding \& He (2004), une Analyse en Composantes Principales (ACP) est menée pour tenter d'établir le nombre de groupes a priori. Les résultats donnent deux facteurs qui expliquent moins de $60 \%$ de la variance expliquée, ce qui est assez faible. Retenir trois facteurs (expliquant 69\%), quatre (76\%) ou cinq facteurs (82\%) permettrait de maximiser la variance expliquée et ainsi de réduire les risques de faible validité.

(2) Une analyse ascendante hiérarchique (avec distance de Ward) est ensuite conduite pour déterminer le nombre de classes pertinent (l'examen visuel du dendrogramme est complété par l'analyse de la chaîne des agrégations). Le pourcentage de variance intra-classe à chaque étape de regroupement suggère une partition en quatre classes qui se justifie en outre, par l'affaiblissement du pourcentage de variabilité (au-delà de 4 classes, il se stabilise à 4\%).

(3) Sur cette base, une analyse d'une partie de l'échantillon français (50\% constitués aléatoirement) est menée par la méthode des nuées dynamiques, et trois, quatre puis cinq classes sont successivement demandées ${ }^{13}$. La même analyse (en 3 temps) est également menée sur l'échantillon anglais (1258 observations). Dans les deux pays, la répartition en trois groupes est peu homogène avec un groupe comprenant environ la moitié de l'effectif (Tableau 3), ce qui s'avère peu discriminant. En outre, les résultats de l'ACP montrent que la variance expliquée par trois groupes est inférieure à $70 \%$ ce qui s'avère insuffisant. La répartition en 5 groupes est plus homogène mais l'effectif d'un des groupes est très faible (moins de 10\%) et les groupes définis sont peu différenciés sur certains avantages, notamment en France (Annexe 4). Avec 4 classes, $80 \%$ de la variance est couverte et les groupes sont plus équilibrés et mieux différenciés, aussi bien au niveau français qu'au niveau anglais.

Globalement, la répartition en 4 classes se révèle donc la plus pertinente, tant du point de vue de la répartition des individus (nombre d'individus suffisamment grand) que de la différence interclasse. Afin de conforter les résultats, l'analyse est reconduite sur les deuxièmes parties des échantillons $(50 \%$ restant $)$ français et anglais. Les résultats convergeant, nous présentons les résultats obtenus sur l'échantillon total.

\footnotetext{
${ }^{13}$ Ces trois solutions sont liés au fait que : 1) une typologie en 2 classes, qui reviendrait à distinguer ceux qui ne donnent jamais d'informations et ceux qui en donnent facilement aurait peu d'intérêt, tant d'un point de vue académique que pratique ; 2) l'ACP fait pencher pour un nombre de classes compris entre 3 et 5 ; 3) l'analyse hiérarchique et la littérature font plutôt pencher pour une typologie en 4 classes, d'où l'intérêt de vérifier si une solution présentant une classe de plus ou de moins sont envisageables ; 4) les solutions supérieures à cinq classes ont peu de sens du fait du nombre souvent limité d'observations dans une des classes au moins (Ray 1982).
} 
Tableau 3 - Répartition en 3, 4 et 5 groupes en France et en Angleterre

\begin{tabular}{ccc|ccc}
\hline & \multicolumn{3}{|c}{ France } & \multicolumn{3}{c}{ Angleterre } \\
\hline 3 groupes & 4 groupes & 5 groupes & 3 groupes & 4 groupes & 5 groupes \\
$33,5 \%$ & $\mathbf{3 2 . 2 \%}$ & $21,6 \%$ & $14,5 \%$ & $\mathbf{3 7 . 3 \%}$ & $9,6 \%$ \\
$16,3 \%$ & $\mathbf{2 1 . 6 \%}$ & $22,6 \%$ & $47,1 \%$ & $\mathbf{1 9 . 9 \%}$ & $24,2 \%$ \\
$50,2 \%$ & $\mathbf{3 4 . 5 \%}$ & $27,8 \%$ & $38,4 \%$ & $\mathbf{3 0 . 9 \%}$ & $23,4 \%$ \\
& $\mathbf{1 1 . 8 \%}$ & $18,2 \%$ & & $\mathbf{1 2 . 0 \%}$ & $24,2 \%$ \\
& & $9,8 \%$ & & & $18,5 \%$ \\
\hline
\end{tabular}

\section{PRESENTATION ET DISCUSSION DES RESULTATS}

\subsection{La typologie (TARED) obtenue : quatre groupes distincts}

D’après nos résultats, les internautes interrogés se distinguent quant aux avantages qu'ils recherchent en échange de leurs données personnelles et quatre groupes sont obtenus dans chaque pays (tableau 4). La typologie trouvée conforte les résultats découverts dans une étude quantitative exploratoire (auteur) menée en France sur 1364 internautes aguerris (dont l'échantillon était plus âgé que celui-ci) où quatre groupes avaient aussi été identifiés. Ceci confère ainsi une certaine validité externe à notre typologie, cette validité étant encore accentuée ici par le fait que la répartition au sein des groupes est sensiblement identique d'un pays à l'autre. Ainsi, les «bienveillants » rassemblent environ un tiers des effectifs (un peu moins en France et un peu plus en Angleterre) tout comme les «négociateurs » qui sont toutefois un peu plus nombreux en France (34.5\% vs. $30.9 \%$ en Angleterre). Les «désintéressés » comptabilisent environ $20 \%$ des effectifs et les réticents $12 \%$. Nous reviendrons sur la signification de ces groupes et sur les - éventuelles - différences France/Angleterre dans la suite de l'article.

Tableau 4 - Répartition des internautes en 4 groupes en France et en Angleterre

\begin{tabular}{clcccc}
\hline Classe & \multicolumn{1}{c}{ Interprétation } & $\begin{array}{c}\text { Effectif en } \\
\text { France }\end{array}$ & $\begin{array}{c}\text { Part en } \\
\text { France }(\%)\end{array}$ & $\begin{array}{c}\text { Effectif en } \\
\text { Angleterre }\end{array}$ & $\begin{array}{c}\text { Part en } \\
\text { Angleterre }(\%)\end{array}$ \\
\hline 1 & Les bienveillants (BIEN) & 648 & $\mathbf{3 2 . 2 \%}$ & 469 & $\mathbf{3 7 . 3 \%}$ \\
2 & Les désintéressés (DES) & 434 & $\mathbf{2 1 . 6 \%}$ & 250 & $\mathbf{1 9 . 9 \%}$ \\
3 & Les négociateurs (NEG) & 695 & $\mathbf{3 4 . 5 \%}$ & 389 & $\mathbf{3 0 . 9 \%}$ \\
4 & Les réticents (RET) & 237 & $\mathbf{1 1 . 8 \%}$ & 150 & $\mathbf{1 2 . 0 \%}$ \\
\hline
\end{tabular}

En s'appuyant sur les avantages recherchés par les individus appartenant à chaque groupe (Annexe 5), il est possible de donner du sens aux quatre classes. Malgré une préférence quasi identique pour les différents bénéfices proposés en France et en Angleterre, des différences apparaissent quant à l'importance de certains avantages. Ainsi, les réticents anglais sont beaucoup plus réticents que leurs homologues français puisque plusieurs notes 
tournent autour de 4,5 (5 étant le maximum) alors qu'elles sont égales ou inférieures à 4 dans l'échantillon français. Dans l'ensemble l'avantage "sécurité" discrimine assez peu les groupes (ce qui est confirmé par un F de 138 pour la France et de 92 pour l'Angleterre) tandis que l'avantage "cadeaux" est celui qui les discrimine le plus (F respectivement de 1183 et 719), et ce, quelle que soit la nationalité de l'individu.

Les Bienveillants (groupe 1) sont caractérisés par des notes basses sur tous les critères (une note de 1 étant le signe d'un fort attrait pour ce type d'avantage). Ils délivrent donc facilement leurs données personnelles, quels que soient les bénéfices proposés. Le fait qu'ils puissent obtenir des garanties (sécurité des données) et des avantages en échange (prix, cadeaux) peut être un élément incitatif mais pas forcément décisif puisqu'ils prennent généralement plaisir à se dévoiler, notamment chez les Anglais. Notons qu'obtenir des informations pertinentes en échange des données est beaucoup moins valorisé dans l'échantillon français (2,07 vs. 1,67 pour les Anglais).

Les Désintéressés (groupe 2) ne sont pas dans une optique d'échange de données intensive. Ils sont surtout contre la personnalisation et les avantages de type «monétaire » (réduction de prix et cadeaux). En résumé, ils ne sont prêts à partager leurs informations que si cela ne les implique pas de façon trop importante et surtout si cela leur permet d'obtenir un meilleur service ou d'avoir des garanties en termes de sécurité. Notons que les Anglais désintéressés valorisent davantage que les Français le fait de pouvoir obtenir des informations pertinentes et le plaisir qu'ils peuvent ressentir mais qu'ils sont particulièrement opposés aux cadeaux pouvant être obtenus en échange.

Les Négociateurs (groupe 3) adoptent une position de donnant-donnant. Ils sont prêts à délivrer des informations personnelles pour des avantages particuliers comme un meilleur prix, un cadeau, une personnalisation des offres, bénéfices qui sont toutefois légèrement moins plébiscités du côté anglais. Ils ne délivrent pas systématiquement leurs données mais ont pris conscience du fait qu'elles représentent une valeur d'échange, qu'ils sont enclins à faire jouer lorsque la transaction proposée les intéresse.

Les Réticents (groupe 4) obtiennent les notes les plus hautes sur tous les avantages recherchés. Leur comportement sera donc le plus souvent un comportement d'évitement puisqu'ils prennent rarement plaisir à délivrer des informations les concernant. Même les garanties affichées par un site en matière de sécurité ont peu de chance de les influencer puisque leur position de principe est de fournir le minimum de renseignements. Ils sont surtout contre la personnalisation qu'ils voient comme le signe d'une identification préalable des goûts des personnes. Notons que les réticents anglais sont particulièrement peu enclins à se dévoiler et qu'ils détestent les réductions de prix et les cadeaux promis en échange. 
Nous confirmons donc l'existence de différences quant aux avantages recherchés en échange du dévoilement, validant ainsi l'hypothèse H1. Par ailleurs, si l'on compare les résultats obtenus pour les deux cultures, on trouve des écarts dans l'importance accordée aux différentes catégories d'avantages, validant ainsi l'hypothèse $\mathbf{H 2}$.

Ces résultats peuvent être utilement comparés aux conclusions issues de la littérature. Nos conclusions confirment tout d'abord une étude quantitative exploratoire du même type menée sur un échantillon français d'internautes adultes (auteur). Plus précisément, nous trouvons à chaque fois une structure des dimensions identique en termes de répartition des individus et de préférence pour les différents avantages proposés. Par ailleurs, on trouve aussi des similitudes avec les résultats de Spiekerman et al. (2001) et Sheehan (2002) qui obtenaient également 4 groupes d'individus. Ainsi, les «fondamentalistes » et les «alarmés » correspondent à nos «réticents » et les «non préoccupés» coïncident avec nos «bienveillants ». Certaines de nos classes se rapprochent aussi de celles obtenues par des chercheurs n'ayant identifié que 3 groupes. Ainsi, les « convenience seekers » de Hann et al (2002) se rapprochent de nos «désintéressés ». Si les catégories extrêmes (fondamentalistesréticents, d'une part, et non préoccupés-bienveillants, d'autre part) semblent relativement stables d'un auteur à l'autre, la différence semble donc s'établir sur les catégories intermédiaires. La nouveauté et l'originalité de la typologie que nous présentons ici est de distinguer les individus prêts à fournir des données si les conditions de sécurité sont respectées (désintéressés) de ceux qui sont dans une véritable optique d'échange (négociateurs). Cela revient alors à scinder la catégorie des «pragmatiques », identifiée par de nombreux auteurs (Cranor, Reagle et Ackerman 1999 ; Jensen, Potts et Jensen 2005 ; Westin 1996), en deux, selon l'aspect sur lequel l'individu se base pour prendre sa décision (sécuritaire ou utilitaire). Il se pourrait donc, comme le signalait Sheehan (2002) que la typologie en 3 classes, établie à l'origine par Westin (1967) à propos des préoccupations de vie privée à travers les médias traditionnels, trouve ses limites quand il s'agit de catégoriser des populations d'internautes et qu'il faille alors plutôt se baser sur une typologie en 4 classes, notamment parce que la catégorie intermédiaire (celle des pragmatiques) ne forme pas un groupe homogène (Jensen et al. 2005). La proximité des résultats entre l'échantillon français et anglais en termes de répartition des individus dans les classes (tableau 4) confirme cette hypothèse. Elle conduit aussi à crédibiliser les résultats obtenus et à valider - au moins provisoirement - la typologie obtenue, ce qui permet d'envisager une description approfondie des profils d'individus appartenant aux classes en termes d'attitudes en matière de RVP (4.2), de comportements habituels (4.3) mais aussi de caractéristiques sociodémographiques et expérientielles (4.4). Nous étudierons également les différences en termes de contexte (4.5). 


\subsection{Les différences d'attitude}

Parmi les éléments attitudinaux (et perceptuels) pouvant discriminer l'appartenance aux classes, on trouve les risques perçus à dévoiler des données personnelles (Pavlou 2003), le niveau de confiance dans le média Internet (Castaneda et Montoro 2007), le niveau de préoccupation pour le RVP (Annexe 1) et la perception du niveau de protection offert par la règlementation (Milberg et al. 1995). Nous y ajoutons l'innovativité (capacité à adopter rapidement des innovations technologiques) en faisant l'hypothèse que les individus au degré d'innovativité élevé, ayant une moins forte aversion au risque (Rogers 2003), seront plus enclins à fournir des données. Les résultats des tests de différences de moyennes confirment que ces éléments ont un effet significatif (au seuil de 1\%) sauf en ce qui concerne l'innovativité qui n'est pas significative du côté anglo-saxon $(\mathrm{p}=0,156)$ (tableaux 5 et 6 ). Du côté français, comme nous l'avions supposé, les Bienveillants se montrent les plus innovatifs et les Réticents le moins. Même si cela n'est pas confirmé dans l'échantillon anglais, l'ordre reste cependant identique suggérant que l'innovativité pourrait influencer la capacité de dévoilement, les plus innovatifs semblant les plus prompts à se dévoiler.

Sans surprise, aussi bien en France qu'en Angleterre, les Bienveillants ont le niveau de confiance dans Internet et le niveau de protection perçue les plus élevés (4,16 et 4,60 en France) ainsi que le niveau de risque perçu et le niveau de préoccupation les plus faibles $(4,59$ et 2,08 en France), au contraire des Réticents qui se sentent les moins en confiance, les moins protégés $(2,80$ et 4,02$)$ et les plus préoccupés (1,68 en France). Ces caractéristiques des Réticents expliquent qu'ils refusent la plupart du temps de délivrer des renseignements, à l'opposé des Bienveillants. Notons que Désintéressés et Négociateurs se distinguent assez peu sur ces critères, les derniers se montrant toutefois plus méfiants et moins rassurés, ce qui les amène à exiger davantage de compensations en échange de leurs données à caractère personnel. Dans l'échantillon anglais, les risques liés au dévoilement sont globalement perçus comme plus élevés et le niveau de protection est jugé plus faible que dans l'échantillon français. Par ailleurs, concernant le niveau de préoccupation, les Désintéressés anglais se rapprochent des Réticents, signe d'une plus grande défiance à ce sujet dans ce pays. Dans l'échantillon français, les Désintéressés se rapprochent des Bienveillants sur cette variable, signe d'une plus grande confiance dans la capacité du législateur à protéger ses citoyens. Dans l'ensemble, nous confirmons l'existence de différences selon l'attitude face au RVP (hormis pour l'innovativité chez les Anglais), validant ainsi - à cette nuance près - l'hypothèse $\mathbf{H 3}$. 
Tableau 5 - Test de moyenne sur les différences de perceptions, d'attitude et de comportement entre les groupes au niveau français

\begin{tabular}{|c|c|c|c|c|c|c|c|c|c|c|c|c|}
\hline \multirow{3}{*}{ Classe } & \multirow{3}{*}{$\begin{array}{l}\text { Effectifs } \\
\text { (France) }\end{array}$} & \multicolumn{5}{|c|}{ Perceptions et attitudes } & \multicolumn{6}{|c|}{ Comportement de dévoilement et de protection } \\
\hline & & & & & & & \multicolumn{5}{|c|}{ Comportement de dévoilement habituel } & \multirow{2}{*}{$\begin{array}{c}\text { Comporte } \\
\text {-ment de } \\
\text { protection } \\
\text { habituel }\end{array}$} \\
\hline & & Innovativité & $\begin{array}{l}\text { perçus au } \\
\text { dévoilement }\end{array}$ & $\begin{array}{c}\text { dans } \\
\text { Internet }\end{array}$ & $\begin{array}{c}\text { Préoccupation } \\
\text { pour le RVP }\end{array}$ & $\begin{array}{c}\text { protection } \\
\text { perçue }\end{array}$ & $\begin{array}{l}\text { Donne sa } \\
\text { véritable } \\
\text { identité }\end{array}$ & $\begin{array}{c}\text { Utilise } \\
\text { pseudo- } \\
\text { nyme }\end{array}$ & $\begin{array}{l}\text { Donne le } \\
\text { minimum }\end{array}$ & $\begin{array}{c}\text { Mensonge } \\
\text { (données } \\
\text { erronées }\end{array}$ & $\begin{array}{l}\mathrm{Ne} \\
\text { répond } \\
\text { pas }\end{array}$ & \\
\hline 1 (Bienveillants) & $\mathrm{N}=648$ & 4,67 & 4,59 & 4,16 & 2,08 & 4,60 & 2,44 & 3,08 & 2,81 & 1,66 & 2,24 & 2,44 \\
\hline 2 (Désintéressés) & $\mathrm{N}=434$ & 4,48 & 4,99 & 3,78 & 1,99 & 4,51 & 2,26 & 3,02 & 2,80 & 1,65 & 2,42 & 2,35 \\
\hline 3 (Négociateurs) & $\mathrm{N}=695$ & 4,37 & 5,02 & 3,56 & 2,02 & 4,33 & 2,24 & 3,13 & 2,75 & 1,80 & 2,40 & 2,42 \\
\hline 4 (Réticents) & $\mathrm{N}=237$ & 4,13 & 5,49 & 2,80 & 1,68 & 4,02 & 1,96 & 3,09 & 2,72 & 1,98 & 2,46 & 2,49 \\
\hline Total & $\mathrm{N}=2014$ & 4,46 & 4,93 & 3,71 & 1,99 & 4,42 & 2,28 & 3,08 & 2,77 & 1,75 & 2,36 & 2,42 \\
\hline $\mathrm{F}$ & & 8,779 & 26,547 & 70,508 & 16,394 & 8,833 & 29,527 & 1,952 & 1,108 & 15,761 & 8,723 & 3,313 \\
\hline Sig. & &, 000 &, 000 &, 000 &, 000 &, 000 &, 000 & 0,119 & 0,344 &, 000 &, 000 & ,019 \\
\hline
\end{tabular}

Tableau 6 - Test de moyenne sur les différences de perceptions, d'attitude et de comportement entre les groupes au niveau anglais

\begin{tabular}{|c|c|c|c|c|c|c|c|c|c|c|c|c|}
\hline \multirow{3}{*}{ Classe } & \multirow{3}{*}{$\begin{array}{c}\text { Effectifs } \\
\text { (Angle- } \\
\text { terre) }\end{array}$} & \multicolumn{5}{|c|}{ Perceptions et attitudes } & \multicolumn{6}{|c|}{ Comportement de dévoilement et de protection } \\
\hline & & & & Conf & & Niveau de & \multicolumn{5}{|c|}{ Comportement de dévoilement habituel } & \multirow{2}{*}{$\begin{array}{c}\text { Comporte } \\
\text {-ment de } \\
\text { protection } \\
\text { habituel }\end{array}$} \\
\hline & & Innovativité & $\begin{array}{c}\text { perçus au } \\
\text { dévoilement }\end{array}$ & $\begin{array}{l}\text { dans } \\
\text { Internet }\end{array}$ & $\begin{array}{c}\text { Préoccupation } \\
\text { pour le RVP }\end{array}$ & $\begin{array}{l}\text { protection } \\
\text { perçue }\end{array}$ & $\begin{array}{c}\text { Donne sa } \\
\text { véritable } \\
\text { identité }\end{array}$ & $\begin{array}{c}\text { Utilise } \\
\text { pseudo- } \\
\text { nyme }\end{array}$ & $\begin{array}{l}\text { Donne le } \\
\text { minimum }\end{array}$ & $\begin{array}{c}\text { Mensonge } \\
\text { (données } \\
\text { erronées }\end{array}$ & $\begin{array}{l}\mathrm{Ne} \\
\text { répond } \\
\text { pas }\end{array}$ & \\
\hline 1 (Bienveillants) & $\mathrm{N}=469$ & 4,51 & 5,19 & 3,93 & 1,78 & 3,87 & 2,55 & 1,91 & 2,66 & 1,57 & 2,23 & 2,46 \\
\hline 2 (Désintéressés) & $\mathrm{N}=250$ & 4,36 & 5,58 & 3,58 & 1,66 & 3,52 & 2,48 & 1,98 & 2,90 & 1,59 & 2,45 & 2,53 \\
\hline 3 (Négociateurs) & $\mathrm{N}=389$ & 4,28 & 5,35 & 3,61 & 1,93 & 3,57 & 2,41 & 1,84 & 2,67 & 1,72 & 2,35 & 2,45 \\
\hline 4 (Réticents) & $\mathrm{N}=150$ & 4,25 & 6,06 & 2,55 & 1,53 & 2,69 & 2,17 & 2,05 & 3,06 & 1,83 & 2,84 & 2,86 \\
\hline Total & $\mathrm{N}=1258$ & 4,38 & 5,42 & 3,60 & 1,77 & 3,57 & 2,45 & 1,92 & 2,76 & 1,66 & 2,38 & 2,52 \\
\hline $\mathrm{F}$ & & 1,745 & 13,588 & 39,651 & 11,592 & 20,629 & 8,881 & 2,853 & 12,954 & 6,851 & 25,382 & 12,777 \\
\hline Sig. & &, 156 &, 000 &, 000 &, 000 &, 000 &, 000 & 0,036 &, 000 &, 000 &, 000 & ,000 \\
\hline
\end{tabular}

En gras, les moyennes inférieures ou supérieures à la moyenne de l'échantillon (au seuil de 5\%)

En gris clair, les moyennes statistiquement supérieures dans l'échantillon français et en gris foncé celles statistiquement supérieures dans l'échantillon anglais 


\subsection{Les différences de comportement}

L'examen des comportements habituels de dévoilement et de protection (tableaux 5 et 6) permet également de faire ressortir des différences intéressantes. En matière de protection face aux utilisations potentiellement frauduleuses de leur profil ${ }^{14}$, la classe la plus protectrice, tant en France qu'en Angleterre, est celle des Réticents qui cherchent ainsi à éviter les menaces potentielles. Négociateurs et Bienveillants ont une conduite similaire, et ce dans les deux pays, probablement parce que leur tendance à se dévoiler - utilitaire pour les premiers et naturelle pour les seconds - les amène à une surexposition aux risques qu'ils peuvent parfois vouloir compenser par une stratégie de défense.

Ces résultats sont confortés par ceux qui concernent les comportements habituellement adoptés par les individus lorsqu'on leur demande de s'identifier en ligne. Si l'utilisation de pseudonymes est la stratégie préférée des internautes français, elle ne discrimine pas l'appartenance aux classes (moy. $=3, \mathrm{p}=0,119$ ) contrairement à l'usage anglais qui est beaucoup plus restreint (moy. $=1,92 ; \mathrm{p}=0,036$ ) mais plus différenciant: les Réticents anglais en sont le plus friands à l'opposé des Négociateurs, avec les Bienveillants et les Désintéressés au comportement proche de la moyenne. Pour la stratégie consistant à fournir le minimum d'informations possibles, les Anglais y ont fréquemment recours alors qu'elle ne discrimine pas les classes côté français. Concernant le mensonge (fourniture de données erronées), on trouve une configuration similaire dans les deux pays, les Réticents étant les plus prompts à utiliser cette stratégie à l'opposé des Bienveillants et Désintéressés (aux comportements à nouveau similaires), les Négociateurs se situant entre les deux, au gré des situations et des avantages susceptibles d'être obtenus en échange. Enfin, les Bienveillants ont tendance à donner volontiers leur véritable identité alors que les Réticents préfèrent souvent ne pas répondre aux requêtes des sites. A noter que, sur ces deux points, les Bienveillants se rapprochent cette fois-ci des Négociateurs avec un comportement souvent intermédiaire.

Nous confirmons alors, puisque les quatre groupes sont différents sur ce critère, que les comportements habituels en matière de RVP (comportements de dévoilement et de protection) influencent le type d'avantages recherchés en échange de la fourniture de données personnelles en ligne, validant ainsi au moins partiellement - puisque certaines habitudes de dévoilement (comme le recours au pseudonyme) n'offrent pas de caractère discriminant -

\section{l'hypothèse $\mathrm{H4}$.}

\footnotetext{
${ }^{14}$ Trois items servent à établir le niveau de protection adopté par l'individu. Des items portant sur l'utilisation d'anti-virus ou de firewalls ont été écartés au profit de questions sur la lecture des chartes et le changement des paramètres du navigateur. Ces items correspondent volontairement à des mesures « avancées », pour conférer de la variance et donc un véritable statut différenciant à cette variable.
} 


\subsection{Le profil expérientiel et sociodémographique des individus de chaque classe}

Les individus des différentes classes présentent des profils distincts en termes d'expérience et d'usage d'Internet ${ }^{15}$ mais aussi de genre, de CSP et de niveau d'étude (tableau 7). Concernant l'expérience en ligne ${ }^{16}$, on constate que la majorité des répondants français ont atteint un niveau intermédiaire (37.9\%) ou avancé $(6.1 \%)$. Les individus réalisant des activités basiques (26\%) sont, soit Négociateurs (28.3\%) soit Réticents (30.0\%), différence significative à $1 \%$. Les individus aux activités intermédiaires sont plutôt Désintéressés tandis que les plus avancés sont plutôt des Bienveillants Ces résultats se retrouvent également dans l'échantillon anglais. Sur le plan de l'ancienneté de l'usage d'Internet, les résultats (significatifs au seuil de 3\%) montrent que les internautes français les plus anciens (plus de 5 ans) appartiennent aux classes 2 et 4 (Désintéressés et Réticents). Leur expérience les a-t-elle conduit à être blasés, pour les premiers, ou au contraire méfiants, pour les seconds, suite à certaines mésaventures ? Il serait intéressant de creuser les motivations liées à ces positions. A noter qu'une différence avec l'échantillon anglais apparait : la non significativité de cette variable «ancienneté Internet », qui ne discrimine pas l'appartenance aux classes, alors que c'est le cas dans l'échantillon français. Une explication possible réside dans le manque de variance sur cette variable dans l'échantillon anglais $(25 \%$ de «novices » versus $75 \%$ d'« experts »). Ce problème pourra être résolu par la réplication de cette étude sur un échantillon plus représentatif de la population. Ceci permettra alors de vérifier de manière robuste le caractère discriminant ou non de cette variable. Nous validons ainsi H5, au moins partiellement, puisque la variable d'usage n'est pas significative dans l'échantillon anglais.

Concernant les aspects sociodémographiques, toutes les variables testées - hormis le type d'habitat - sont significatives, et ce, aussi bien en France qu'en Angleterre, ce qui apporte une solidité à ces résultats et valide ainsi H6. Plus précisément, les hommes français (39.8\% de l'effectif) sont particulièrement présents (48.8\%) dans la classe 2 (Désintéressés) alors que les femmes appartiennent majoritairement aux trois autres catégories. Dans l'échantillon anglais, les femmes sont surtout Négociateurs, indiquant ainsi une pratique mature de 1'Internet. Au niveau de la CSP, les employés sont majoritairement Bienveillants (27\%) tandis que les sans-emploi sont plutôt Négociateurs (11.4\%). Les étudiants sont, soit Désintéressés (61.3\%), soit Réticents (58.6\%). En termes de niveau d'étude, les répondants de

\footnotetext{
${ }^{15}$ Nous ne présentons pas de résultat pour l'âge, l'échantillon présentant peu de variance puisque les répondants ont entre 15 et 25 ans. Les analyses menées sur une catégorisation de cette variable en 2 (15-20 puis 21-25) ou 3 catégories $(15-18,19-21,22-25)$ confirment sa non significativité pour les français comme pour les anglais.

${ }^{16}$ Pour cette variable, 3 groupes sont créés : les individus réalisant des activités basiques (ex : consultation d'emails et utilisation de moteurs de recherche) qualifiés de novices, ceux réalisant des activités intermédiaires (ex : téléphone en ligne, maintien d'un site) qualifiés d'intermédiaires et ceux réalisant des activités avancées/ évoluées (entretien d'un réseau social) qualifiés d'experts.
} 
niveau supérieur à Bac +2 ou 3 (18.8\%) sont surtout présents chez les Désintéressés (26.2\%) et sous-représentés (13.9\%) dans la classe des Négociateurs, alors que les répondants dont le niveau d'études est inférieur ou égal au Bac (au total 40.1\%) sont surreprésentés dans la classe des Négociateurs (46.2\%). Ces résultats se confirment dans l'échantillon anglais.

Tableau 7 - Chi 2 sur les variables sociodémographiques et d'expérience en ligne

\begin{tabular}{|c|c|c|c|}
\hline $\begin{array}{c}\text { Variables } \\
\text { signalétiques }\end{array}$ & $\begin{array}{l}\text { Résultats } \\
\text { (France) }\end{array}$ & $\begin{array}{c}\text { Résultats } \\
\text { (Angleterre) }\end{array}$ & Commentaires \\
\hline $\begin{array}{l}\text { Genre } \\
(\mathrm{H}, \mathrm{F})\end{array}$ & $\begin{array}{c}\chi^{2}=21,277 \\
\mathrm{ddl}=3 ; \mathrm{p}=0,000\end{array}$ & $\begin{array}{c}\chi^{2}=17,374 \\
\mathrm{ddl}=3 ; p=0,000\end{array}$ & $\begin{array}{c}\text { Relation significative } \\
\text { Les femmes sont plutôt négociateurs }\end{array}$ \\
\hline $\begin{array}{l}\text { Habitat } \\
\text { (urbain, rural) }\end{array}$ & $\begin{array}{c}\chi^{2}=10,293 \\
\mathrm{ddl}=6 ; \mathrm{p}=0,113\end{array}$ & $\begin{array}{c}\chi^{2}=10,287 \\
\mathrm{ddl}=6 ; \mathrm{p}=0,114\end{array}$ & Relation non significative \\
\hline $\begin{array}{l}\text { CSP } \\
\text { (étudiants, employés, } \\
\text { sans emploi, autres) }\end{array}$ & $\begin{array}{c}\chi^{2}=23,591 \\
\mathrm{ddl}=9 ; \mathrm{p}=0,005\end{array}$ & $\begin{array}{c}\chi^{2}=41,239 \\
\mathrm{ddl}=9 ; p=0,0045\end{array}$ & $\begin{array}{c}\text { Relation significative } \\
\text { Les sans-emplois sont majoritairement } \\
\text { des négociateurs }\end{array}$ \\
\hline $\begin{array}{l}\text { Niveau d'étude } \\
\text { (bac; deug-licence, } \\
\text { master ou doctorat) }\end{array}$ & $\begin{array}{c}\chi^{2}=42,298 \\
\mathrm{ddl}=6 ; \mathrm{p}=0,000\end{array}$ & $\begin{array}{c}\chi^{2}=18,030 \\
\mathrm{ddl}=6 ; p=0,006\end{array}$ & $\begin{array}{l}\text { Relation significative } \\
\text { Les négociateurs sont peu éduqués } \\
\text { tandis que les désintéressés ont un } \\
\text { niveau d'études supérieur à } \mathrm{Bac}+2\end{array}$ \\
\hline $\begin{array}{l}\text { Ancienneté d'usage } \\
\text { d'Internet } \\
\text { (+ ou - de } 5 \text { ans) }\end{array}$ & $\begin{array}{c}\chi^{2}=9,204 \\
\mathrm{ddl}=3 ; \mathrm{p}=0,027\end{array}$ & $\begin{array}{c}\chi^{2}=2,031 \\
\mathrm{ddl}=3 ; \mathrm{p}=0,566\end{array}$ & $\begin{array}{l}\text { Relation significative (France) mais } \\
\text { non significative (Angleterre) } \\
\text { Les désintéressés et les réticents sont } \\
\text { connectés depuis longtemps }\end{array}$ \\
\hline $\begin{array}{l}\text { Expérience en ligne } \\
\text { (novices, intermé- } \\
\text { diaires, experts) }\end{array}$ & $\begin{array}{c}\chi^{2}=17,203 \\
\mathrm{ddl}=6 ; \mathrm{p}=0,009\end{array}$ & $\begin{array}{c}\chi^{2}=12,691 \\
\mathrm{ddl}=6 ; \mathrm{p}=0,048\end{array}$ & $\begin{array}{l}\text { Relation significative } \\
\text { Les réticents font surtout des activités } \\
\text { basiques et les désintéressés des } \\
\text { activités avancées }\end{array}$ \\
\hline
\end{tabular}

\subsection{L'effet de la situation}

Nous avions également fait l'hypothèse d'un effet du contexte dans lequel les données sont sollicitées (niveau de confiance dans l'interlocuteur et type de données demandées) sur le type d'avantages recherchés. On trouve effectivement des différences entre les classes, en ce qui concerne le niveau de confiance vis-à-vis des différents types d'interlocuteurs possibles (tableau 8). Quel que soit l'interlocuteur (organisme public, entreprise réputée, entreprise familière, entreprise inconnue ou association), la hiérarchie est toujours la même, et ce dans les deux pays. Les Bienveillants sont ceux qui ont le niveau de confiance le plus élevé (une note proche de 1 indiquant une confiance totale), suivis respectivement par les Désintéressés et les Négociateurs. Les Réticents sont, de loin, ceux qui ont le niveau de confiance le plus faible (le niveau 5 correspondant à une méfiance totale). On remarque également que les replis les plus importants concernent les requêtes d'informations émises par les entreprises inconnues mais aussi par celles qui, bien que connues, n'ont pas de lien antérieur avec l'internaute (entreprise réputée, non familière). Il est ainsi possible de conclure qu'en matière de vie privée, la familiarité de l'internaute avec le site est un facteur plus incitatif que la seule réputation ou notoriété. Le gouvernement et les associations obtiennent des notes assez faibles 
(respectivement 3,13 et 3,26 en France), ce qui confirme l'impact du type de site et l'importance du lien antérieur avec ce site. Cet aspect situationnel de la confiance semble être un des éléments les plus différenciateurs, notamment au niveau anglais où les quatre groupes sont très éloignés les uns des autres, signe de l'importance des "circonstances" dans lesquelles les informations sont demandées et notamment ici du type d'organisation à l'origine de la sollicitation. Ce caractère différenciant de la confiance accordée à l'interlocuteur se retrouve au niveau de la confiance «globale » accordée au média Internet qui discrimine fortement les quatre groupes, notamment les Réticents (les moins confiants) - quelle que soit la nationalité de l'individu - mais aussi les Bienveillants et les Négociateurs, et ce surtout en France.

En résumé, les Bienveillants sont les plus enclins à dévoiler des données personnelles sur Internet, au contraire des Réticents. Ceci se retrouve, notamment, à travers deux comportements phares : donner sa véritable identité et fournir des données à une entreprise réputée mais non familière. Ainsi, une entreprise réputée qui souhaite établir un premier contact avec des prospects risque de les voir mentir ou ne pas donner leur véritable identité.

\section{Tableau 8 - Test de moyenne sur le niveau de confiance selon l'interlocuteur}

\begin{tabular}{|c|c|c|c|c|c|c|c|c|c|c|}
\hline \multirow[t]{2}{*}{$\begin{array}{l}\text { Interlocuteurs } \\
\text { Classes }\end{array}$} & \multicolumn{2}{|c|}{$\begin{array}{l}\text { Le gouver- } \\
\text { nement }\end{array}$} & \multicolumn{2}{|c|}{$\begin{array}{l}\text { une entreprise } \\
\text { réputée }\end{array}$} & \multicolumn{2}{|c|}{$\begin{array}{l}\text { une entreprise } \\
\text { familière }\end{array}$} & \multicolumn{2}{|c|}{$\begin{array}{l}\text { une entreprise } \\
\text { inconnue }\end{array}$} & \multicolumn{2}{|c|}{ une association } \\
\hline & FR & GB & FR & GB & FR & GB & FR & GB & FR & GB \\
\hline 1 (Bienveillants) & 2,96 & 3,13 & 2,79 & 2,60 & 2,40 & 2,44 & 4,00 & 4,03 & 3,10 & 3,21 \\
\hline 2 (Désintéressés) & 3,04 & 3,46 & 3,13 & 3,02 & 2,71 & 2,82 & 4,17 & 4,42 & 3,20 & 3,42 \\
\hline 3 (Négociateurs) & 3,25 & 3,58 & 3,15 & 3,04 & 2,74 & 2,93 & 4,20 & 4,24 & 3,34 & 3,57 \\
\hline 4 (Réticents) & 3,43 & 4,33 & $\mathbf{3 , 5 9}$ & 3,88 & 3,14 & 3,80 & 4,44 & 4,62 & $\mathbf{3 , 5 3}$ & 4,15 \\
\hline Total & 3,13 & 3,47 & 3,08 & 2,96 & 2,68 & 2,80 & 4,17 & 4,24 & 3,26 & 3,48 \\
\hline Sig. & \multicolumn{2}{|c|}{, 000} & \multicolumn{2}{|c|}{, 000} & \multicolumn{2}{|c|}{, 000} & \multicolumn{2}{|c|}{, 000} & \multicolumn{2}{|c|}{, 000} \\
\hline
\end{tabular}

En gras, les moyennes inférieures ou supérieures à la moyenne de l'échantillon (au seuil de 5\%)

Le deuxième élément lié à la situation que nous souhaitions tester concerne le type de données demandées, l'objectif étant d'évaluer d'éventuels écarts entre les renseignements que les individus appartenant à ces classes acceptent ou au contraire refusent de fournir sur un site marchand. Les résultats (graphiques 1 et 2) confirment que les réticents sont systématiquement les moins enclins à délivrer des données personnelles alors que les bienveillants sont les plus prompts à se dévoiler, les catégories intermédiaires se situant à chaque fois entre les deux, et ce, quelle que soit la nationalité. Par ailleurs, alors que tous les individus considèrent le même types de données comme peu sensibles (nom, âge, nationalité et goûts) ou au contraire très sensibles (données financières, médicales, judiciaires et 
biométriques), la sensibilité relative de chaque type diffère selon les groupes, en contradiction avec les résultats trouvés par Cranor, Reagle et Ackerman (1999) mais validant ainsi H7.

Graphique 1 - Types de données fournies selon les groupes en Angleterre

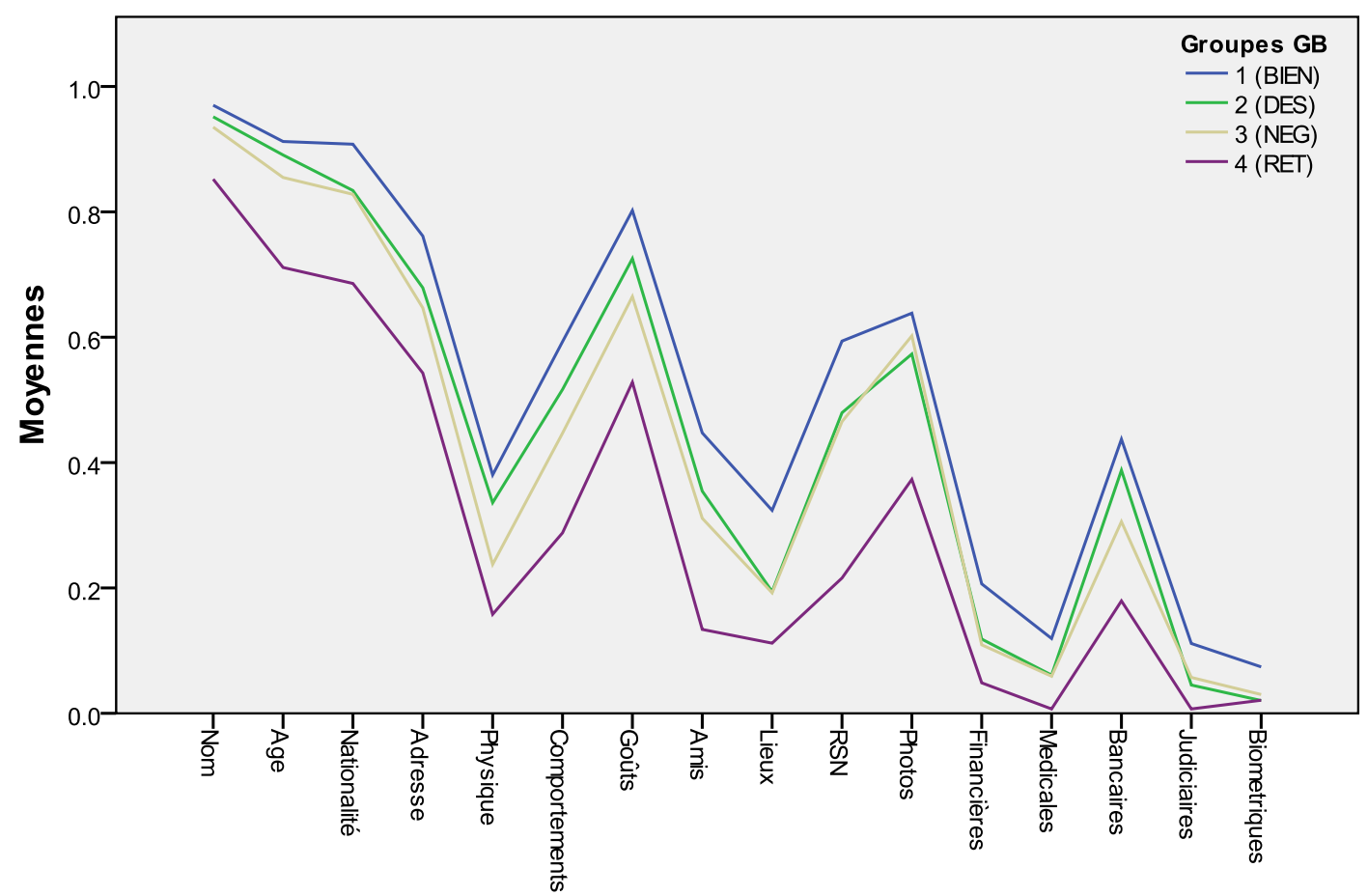

Types de données

Graphique 2 - Types de données fournies selon les groupes en France

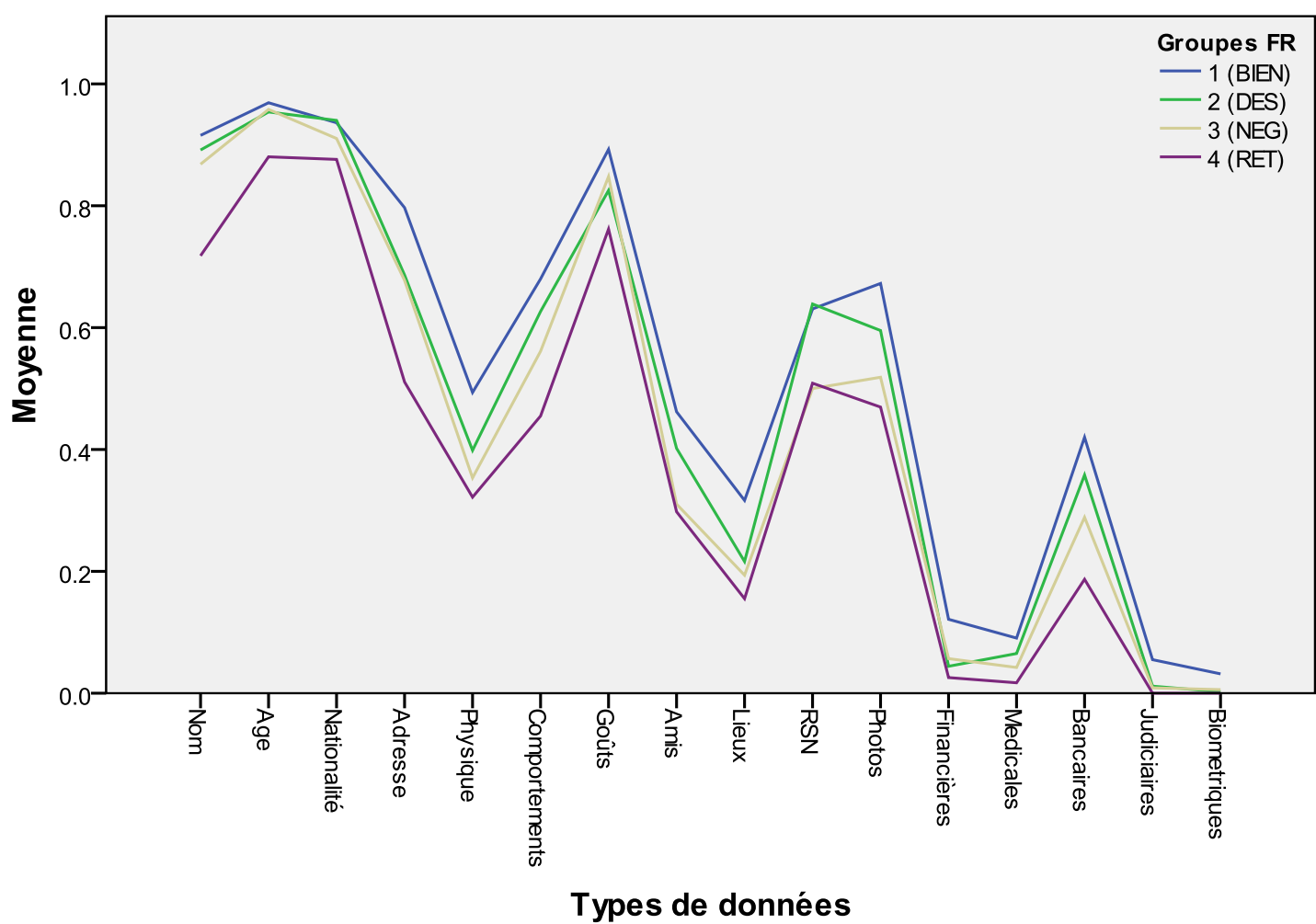


Les différences de comportement entre les groupes sont plus importantes dans l'échantillon anglais et certains types de données, significativement discriminants en Angleterre, ne le sont pas en France (e.g. amis, goûts/opinions, informations données sur les RSN). A noter que les données biométriques, judiciaires, médicales et financières discriminent peu les groupes, la plupart des individus s'avérant peu enclins à partager ce types d'informations, et ce, quelle que soit la nationalité. Pour les catégories 'Désintéressés' et 'Négociateurs', les écarts les plus importants concernant les informations données sur les RSN (pour l'échantillon français) et les goûts (pour l'échantillon anglais).

\subsection{Comparaison interculturelle et synthèse des résultats}

Dans une optique de synthèse, le tableau 9 permet de récapituler, pour chaque classe identifiée, les principaux avantages recherchés (en comparaison de ceux préférés dans les autres classes) ainsi que les valeurs attitudinales et comportementales les plus représentatives, de manière à repérer les différences majeures entre les classes.

\section{Tableau 9 - Récapitulatif des caractéristiques de chacune des classes}

\begin{tabular}{|c|c|c|c|}
\hline Classes & $\begin{array}{c}\text { Principaux avantages recherchés } \\
\text { (en comparaison } \\
\text { avec les autres classes) }\end{array}$ & $\begin{array}{l}\text { Variables descriptives } \\
\text { (tendances) }\end{array}$ & $\begin{array}{l}\text { Attitudes et } \\
\text { Comportements } \\
\text { (tendances) }\end{array}$ \\
\hline Les & information et réseau (GB) & & - confiance élevée \\
\hline Bienveillants (1) & $\begin{array}{l}\text { réductions de prix et gain de temps } \\
\text { (France) }\end{array}$ & - employés & $\begin{array}{l}\text { - donnent souvent leur } \\
\text { véritable identité }\end{array}$ \\
\hline $\begin{array}{l}\text { Les } \\
\text { Désintéressés (2) }\end{array}$ & $\begin{array}{l}\text { bénéficier de meilleurs services et } \\
\text { d'un accès sécurisé } \\
\text { (personnalisation, cadeaux et bais-se } \\
\text { de prix ont un effet repoussoir) }\end{array}$ & $\begin{array}{l}\text { - hommes } \\
\text { - niveau d'études }>\text { Bac }+3 \\
\text { - forte expérience d'Inter- } \\
\text { net, activités avancées }\end{array}$ & $\begin{array}{l}\text { - faible tendance à } \\
\text { mentir }\end{array}$ \\
\hline Les & & - sans emploi & \\
\hline Négociateurs (3) & réductions de prix et cadeaux & $\begin{array}{l}\text { - niveau d'études }<\text { Bac } \\
\text { - activités basiques }\end{array}$ & - les moins préoccupés \\
\hline Les Réticents (4) & $\begin{array}{l}\text { aucun } \\
\text { (mais la sécurité est un plus) }\end{array}$ & $\begin{array}{l}\text { - forte expérience d'Inter- } \\
\text { net, activités basiques }\end{array}$ & $\begin{array}{l}\text { - confiance faible et } \\
\text { forte préoccupation } \\
\text { - tendance à mentir ou } \\
\text { à ne pas répondre }\end{array}$ \\
\hline
\end{tabular}

D’après les résultats de cette recherche, les éléments qui discriminent le plus le type d'avantages recherchés en échange du dévoilement, et ce quelle que soit la nationalité de l'internaute (mais de manière plus marquante en France), sont la confiance dans Internet et les risques perçus au dévoilement (Graphique 3). Ainsi, les individus qui ont le niveau de confiance le plus élevé et le niveau de risque perçu le plus faible sont ceux qui se dévoilent le plus volontiers (Bienveillants) tandis qu'à l'opposé ceux qui se dévoilent le moins (Réticents) ont le niveau de confiance le plus faible et les risques perçus les plus élevés. 
Graphique 3 - Comparaison France / Angleterre sur l'ensemble des variables attitudinales et comportementales (valeurs Max. et Min.)

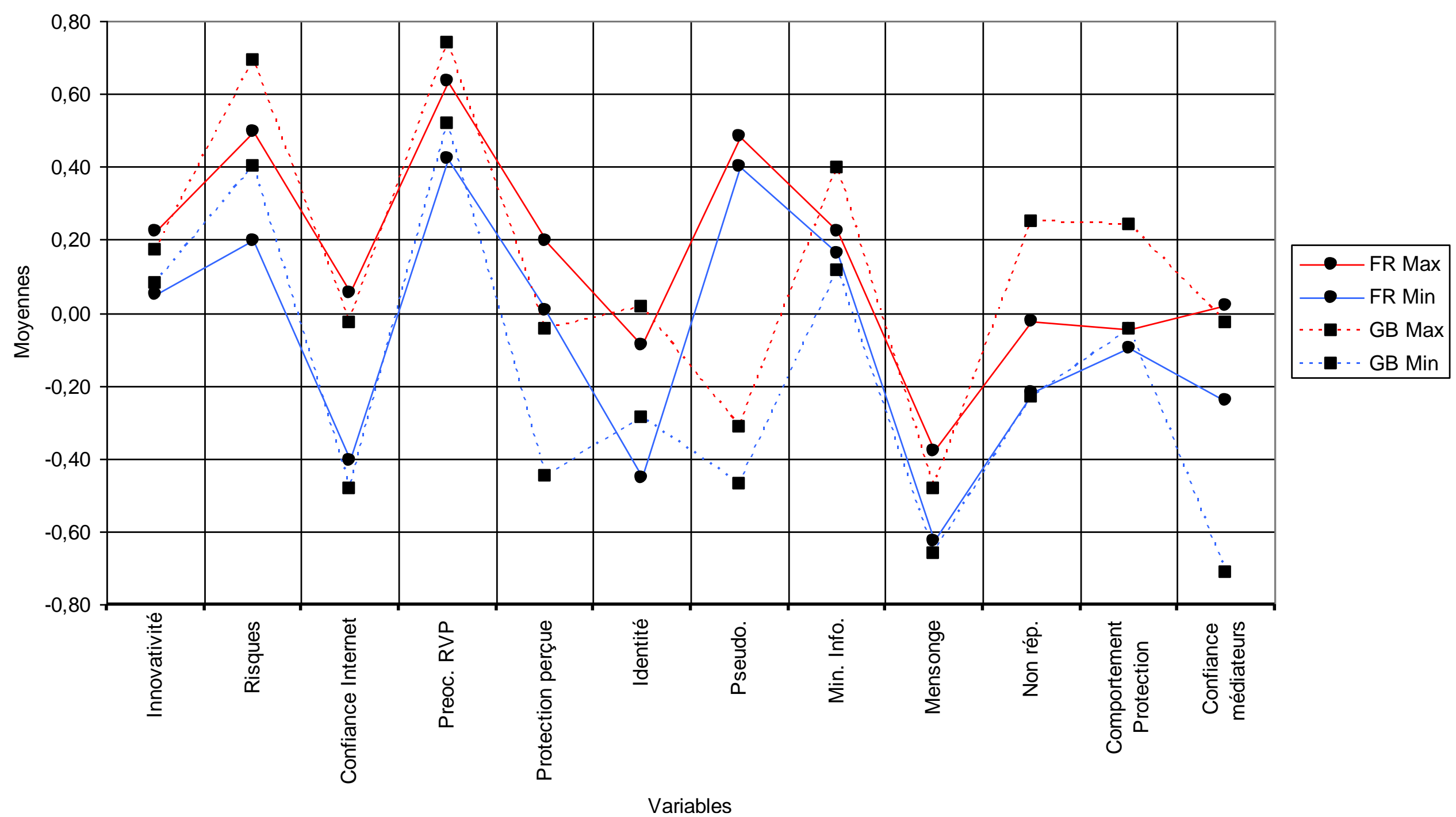


Dans les deux cultures, les Désintéressés et les Négociateurs sont les groupes les plus proches, qui se différencient toutefois par leur tendance au mensonge (en France) et par leur préoccupation pour le RVP (en Angleterre). Les Désintéressés ont peu recours au mensonge (leur comportement est semblable aux Bienveillants dans ce domaine) alors que les Négociateurs y ont recours quand les bénéfices sont intéressants mais qu'ils perçoivent trop de risques à fournir leur véritable identité. Les Négociateurs anglais s'avèrent moins préoccupés par le RVP que les trois autres groupes de l'échantillon anglais (tout en l'étant plus que leurs homologues français), et ce, même si les risques perçus à se dévoiler sont plus élevés que le niveau de risque perçu en France, ce qui va dans le sens des résultats de Dinev et al. (2006). Ceci amène les Réticents anglais à des comportements plus "extrêmes" que leurs homologues français qui consistent à moins répondre aux requêtes des sites et à se protéger davantage. Globalement, les jeunes internautes français se sentent mieux protégés que les Anglais mais ont davantage recours au pseudonyme. Ce recours au pseudonyme, même s'il est moindre en Angleterre, semble s'être démocratisé et ne constitue dès lors plus un élément de différenciation entre les groupes. L'ensemble des résultats (graphique 3 et tableau 10) permet de conclure en l'existence de similarités et de différences dans les deux échantillons, ce qui va à la fois dans le sens de disparités culturelles sur certains aspects et d'universalisation des pratiques pour d'autres (par exemple en ce qui concerne le mensonge). Toutefois, les disparités semblent plus fortes que les similarités en ce qui concerne les comportements (cela est moins vrai pour les attitudes qui se différencient uniquement par des niveaux différents), ce qui tendrait à contredire - en partie au moins - la prédiction de Scott Campbell d'une « disparition des différences nationales à long terme ».

\section{Tableau 10 - Comparaison des résultats selon la culture}

\begin{tabular}{cll}
\hline Variables comparées & \multicolumn{1}{c}{ France } & \multicolumn{1}{c}{ Angleterre } \\
\hline Avantages recherchés & $\begin{array}{l}\text { Les Français valorisent davantage le gain } \\
\text { de temps, l'obtention de services ou } \\
\text { d'avantages, le fait d'entretenir un réseau } \\
\text { (réticents) et l'octroi de baisse de prix } \\
\text { (chez les négociateurs et les réticents). }\end{array}$ & $\begin{array}{l}\text { Les Anglais valorisent surtout l'obtention } \\
\text { d'informations pertinentes et le plaisir de } \\
\text { participer/se dévoiler. }\end{array}$ \\
\hline Variables attitudinales & $\begin{array}{l}\text { Les Français se sentent davantage } \\
\text { protégés. }\end{array}$ & $\begin{array}{l}\text { Les Anglais se sentent préoccupés et } \\
\text { perçoivent davantage de risques à se } \\
\text { dévoiler. Ils se sentent peu protégés par la } \\
\text { législation. }\end{array}$ \\
\hline $\begin{array}{c}\text { Variables } \\
\text { comportementales }\end{array}$ & $\begin{array}{l}\text { Les Français recourent régulièrement et } \\
\text { dès que possible au pseudonyme. }\end{array}$ & $\begin{array}{l}\text { Globalement, les Anglais cherchent à } \\
\text { limiter les informations qu'ils donnent sur } \\
\text { eux. }\end{array}$ \\
\hline $\begin{array}{c}\text { Variables } \\
\text { contextuelles }\end{array}$ & $\begin{array}{l}\text { Les Français ont davantage confiance } \\
\text { dans leur gouvernement et dans les } \\
\text { associations. }\end{array}$ & $\begin{array}{l}\text { Les Anglais valorisent davantage les } \\
\text { entreprises uniquement réputées. }\end{array}$ \\
\hline
\end{tabular}




\section{IMPLICATIONS, LIMITES ET VOIES DE RECHERCHE FUTURES}

\subsection{Apports théoriques}

D’un point de vue théorique, notre étude confirme l'existence de disparités au sein de la population concernant la prédisposition au dévoilement de données personnelles dans le cadre du e-commerce. Comme certains auteurs l'avaient prédit (Sheehan 2002), 4 groupes se dessinent, des plus prompts au dévoilement (les Bienveillants) aux plus opposés (les Réticents), en passant par deux catégories intermédiaires : les Désintéressés, très attachés à la sécurisation des données et les Négociateurs, très attentifs aux bénéfices qu'ils peuvent obtenir en échange. Nos résultats confirment aussi que les internautes peuvent être regroupés en différentes catégories et que cette appartenance permet ensuite de prédire les attitudes et les comportements qui devraient en découler. Notre typologie se distingue des précédents travaux sur plusieurs aspects: 1) le contexte européen de l'étude ; 2) la comparaison interculturelle (France vs. Angleterre) qui confère davantage de validité externe aux résultats ; 3) la distinction de deux catégories intermédiaires qui diffèrent par l'aspect sur lequel l'individu focalise majoritairement son attention (sécuritaire vs. utilitaire). Dès lors, si nos conclusions donnent des résultats similaires à certaines études existantes, les compléments et variations démontrées dans cette étude constituent une extension certaine de la littérature.

Du fait de l'internationalisation de l'usage d'Internet - et de ses conséquences - il est de plus en plus important de considérer les différences culturelles dans la perception du RVP. Ce travail est le premier à comparer deux populations issues d'un même continent, mais de cultures sensiblement différentes (Hofstede 2001). Les études passées menées dans plusieurs pays, tout comme celle présentée ici, montrent des différences dans la taille des classes selon les pays étudiés (e.g. Hann et al. 2002, Ortega Egea et al. 2007) ce qui confirme que les facteurs structurels et culturels influencent les comportements. Toutefois, la littérature - et notre étude le confirme - montre également des similarités aussi bien dans la configuration des groupes que dans les comportements et surtout les attitudes qui en découlent.

Enfin, si la nature contextuelle d'Internet présente aujourd'hui un challenge majeur, l'utilisation de typologies comme celle présentée ici peut être un moyen de mieux répondre à cet enjeu (Sheehan 2002). Nos résultats confirment en effet l'importance du contexte dans l'étude d'Internet (Schoeman 1984) et de la vie privée (Sheehan 2002), d'une part vue la part importante des catégories intermédiaires (56,1\% en France et 50,8\% en Angleterre) qui se décident davantage selon le contexte que d'après des convictions personnelles et, d'autre part, vu l'effet différenciant de variables de contexte comme le type d'interlocuteur et de données demandées. Ceci confirme que «les individus cherchent un équilibre leur permettant de contrôler leur vie privée tout en participant au monde extérieur » ((McLean, 1995, p30). 


\subsection{Apports et implications managériales}

La collecte de données personnelles n'est ni une démarche isolée ni une fin en soi, mais un besoin de connaissance du client motivé par un souci de personnalisation de l'offre et de la relation. Ainsi, quand suite à une visite sur son site pour la recherche d'un voyage, Expédia - à travers son programme Alertes Voyages - envoie un email personnalisé au nom et prénom du client présentant la meilleure offre pour le produit recherché, la démarche est gagnant-gagnant. L'entreprise accroit ses chances de vendre le produit et le client bénéficie d'une offre personnalisée sans pour autant avoir le sentiment d'une intrusion dans sa vie privée puisqu'il peut contrôler (en termes de contenu et de fréquence) les messages publicitaires reçus. Cet effort de personnalisation est d'autant plus bénéfique que les consommateurs apprécient d'être traités en client unique ou spécial (Chelleppa et Shivendu 2007).

Les sites souhaitant mieux connaître leurs clients, de manière à mettre en œuvre un système d'information et une politique relationnelle efficaces, doivent donc réfléchir aux moyens d'inciter les internautes à se dévoiler tout en conservant la confiance nécessaire à l'établissement et au prolongement de la relation. La meilleure façon d'y parvenir consiste, comme dans le cas d'Expédia, à offrir un service à forte valeur à son client. Ce service encourage l'internaute à confier ses données personnelles puisqu'il y a un gain net à l'échange (i.e. plus à gagner qu'à perdre). Une autre solution consiste à imaginer, à l'instar de ce qui se pratique dans les questionnaires d'enquêtes ${ }^{17}$, un dispositif graduel de collecte des informations - des moins sensibles (nom, prénom, email voire adresse postale) aux plus privées (loisirs, situation familiale et professionnelle par exemple) - au fur et à mesure que la relation entre le e-commerçant et l'acheteur évolue. On pourrait en effet se contenter, lors du premier contact, de récolter des informations strictement nécessaires à la transaction (les autres renseignements étant placés en champs facultatifs) puis adapter le formulaire au fur et à mesure de l'évolution de la relation et de la prédisposition du client au dévoilement de ses données personnelles, en y incluant des questions plus personnelles ou en les rendant obligatoires. La première étape permettrait d'identifier les individus les plus prompts à l'échange (désintéressés et bienveillants). Lors des visites suivantes, la sollicitation de données pourrait être adaptée au profil de l'individu et, soit se contenter d'identifier l'individu (cas des réticents), soit aller plus loin en posant de nouvelles questions plus précises et plus privées (cas des bienveillants). Cette recherche souligne donc l'intérêt de recourir à la théorie de l'engagement (Beauvois et Joulé 1981, Kiesler 1971) dans les recherches sur ce thème.

\footnotetext{
${ }^{17}$ Une telle stratégie est connue sous le terme d'engagement et sous l'expression anglaise de «foot in the door » : elle consiste à obtenir du répondant qu'il réponde à une première sollicitation très courte (par exemple répondre à une question), ce premier engagement l'amenant à répondre plus souvent de manière positive aux sollicitations suivantes.
} 
D'un point de vue managérial, la mise en évidence de différentes classes suggère que la question de l'échange de données personnelles dans le cadre du e-commerce doit être abordée avec nuance. A l'évidence, toute solution générale pour contrer les craintes relatives au respect de la vie privée en ligne aura peu de chance de fonctionner. Les sites devront donc chercher à déterminer à quels segments appartiennent leurs différents publics, afin d'adapter au mieux leurs demandes de renseignements aux attentes des catégories ainsi identifiées. Si peu de problèmes se posent pour les individus peu préoccupés par l'exploitation de leurs données (les Bienveillants), l'essentiel de la réflexion devra être consacrée à la manière de convaincre les internautes appartenant aux trois autres segments. Les solutions seront notamment différenciées selon que l'individu se montre concerné par les questions de sécurité (Désintéressé) ou envisage une approche plus pragmatique de l'échange (Négociateur).

Les Désintéressés sont assez peu enclins à se dévoiler. Les avantages de type personnalisation, baisse de prix, argent et cadeaux proposés en échange de l'information ont donc peu de prise sur leur décision. De fait, c'est comme si ce type d'avantage leur faisait prendre conscience du rapport coûts/bénéfices de l'échange et qu'ils se sentaient, soit lésés, soit dévalorisés par ce “ marchandage ". Face à ce type d'individus, à leurs attentes en matière de sécurité, l'entreprise devra principalement démontrer les efforts qu'elle déploie pour offrir le maximum de garanties à l'internaute. Il s'agira donc de mettre en avant, sur la page d'accueil et sur celle contenant le formulaire, le lien pointant vers la charte de confidentialité ainsi que les logos liés à la sécurisation des transferts de données et des paiements.

Les Réticents, opposés par nature à toute diffusion de leurs informations personnelles, n'accepteront de se dévoiler que dans un nombre très limité de circonstances, principalement quand ils n'ont pas la possibilité de faire autrement (par exemple à l'occasion d'un achat de dernière minute). Il est donc essentiel, pour ce type d'individu, non seulement de faciliter le passage à l'acte (i.e. le rassurer par tout signe manifestant le souci de garantir la confidentialité des données dévoilées et la sécurité des transactions) mais également, d'éviter tout " incident" susceptible de le faire revenir sur sa décision. Cela passera notamment par la brièveté et la simplicité du processus de remplissage (peu de champs à remplir, un maximum de champs facultatifs, des réponses automatisées) et par l'absence de questions sensibles.

Inciter les Négociateurs à répondre parait simple de prime abord dans la mesure où ils acceptent assez facilement de dévoiler des renseignements personnels pour peu qu'ils y voient un intérêt. La difficulté réside toutefois principalement dans le choix du type et du niveau de bénéfices à proposer en contrepartie, la valeur de l'échange (rapport coûts/ bénéfices) pouvant être perçue différemment par chaque internaute. Il est, cependant, possible d'imaginer un système incitatif permettant à chacun de choisir le type de contrepartie en échange des 
informations délivrées. Cette solution permettrait de prendre en compte, de manière ouverte et souple, les différents avantages recherchés par ces individus, sans risque de leur faire une offre inadaptée. Un système de points à échanger (tel que le proposent d'ores-et-déjà des sites comme Maximiles ou C-kado) contre, au choix, de l'argent, un bon de réduction ou un cadeau permettrait de couvrir un maximum d'avantages recherchés par ce type d'individus.

Enfin, si les Bienveillants sont généralement favorables à l'idée de livrer leurs données personnelles sur les sites qu'ils visitent, la majorité d'entre eux souhaiteraient cependant pouvoir préciser l'utilisation autorisée des informations ainsi divulguées. Il est dès lors préférable de prévoir des procédures leur permettant d'indiquer eux-mêmes leurs souhaits (cases à cocher) plutôt que de pré-cocher les cases, au risque de les déstabiliser.

En résumé, les entreprises souhaitant obtenir de la part de leurs prospects/clients des données complètes, justes et à jour pour bénéficier d'un système d'information efficace doivent prendre en compte les multiples attentes de ces derniers et s'y adapter. Certaines firmes semblent d'ores-et-déjà avoir compris et intégré les préoccupations du consommateur à l'égard du respect de la vie privée, telles qu'elles ont pu être confirmées à travers cette étude. Toutefois, autant les mesures visant à rassurer les visiteurs quant à la sécurité et la confidentialité des données sont pléthores - sans pour autant garantir une efficacité probante (e.g. Arcand et Nantel 2005, Kimery et McCord 2002) - autant celles qui concernent la simplicité du processus de remplissage et surtout celles favorisant le choix du type de compensation sont rares. Il semble donc qu'il y ait, à ce niveau, une véritable marge de manœuvre pour les managers, d'autant plus intéressante qu'elle a été peu exploitée jusqu'ici. De même, alors que la personnalisation des offres devient courante, pourquoi ne pas imaginer de «personnaliser » aussi la procédure de collecte et, ce faisant, le formulaire de données ? Cette personnalisation gagnerait à être axée, non pas tant sur les caractéristiques sociodémographiques de l'individu (même si certaines jouent un rôle important en matière de préoccupation pour le respect de la vie privée), que sur ses attentes, tant éthiques que relationnelles. Or, jusqu'à présent, la personnalisation est surtout adaptée au profil et aux goûts et préférences des individus. De même, elle se fait après avoir identifié l'individu, ce qui n'est pas sans poser de problèmes liés au respect de l'intimité de chacun (Chellappa et Shivendu 2007). Pourquoi, dès lors, ne pas imaginer un dispositif de personnalisation sans identification? Une personnalisation basée sur le rôle, la mission, la fonction, le profil de l'individu sans qu'il soit pour autant utile de disposer de son identité. Des solutions allant 
dans ce sens existent déjà, notamment sur le web: de l'auto-segmentation ${ }^{18}$ à l'anonymisation ${ }^{19}$, en passant par le filtrage collaboratif (Raghu et al. 2001) popularisé par Amazon («les gens qui ont acheté ce produit ont aussi aimé les produits suivants ») et la personnalisation sur le poste client (client-side personalization) (Cassel et Wolz 2001), voie la plus originale mais la plus délicate à mettre en œuvre (Belleil 2007). Ces systèmes permettent de tirer profit des avantages liés à la personnalisation (pertinence des contenus, gain de temps, qualité de l'information ...) sans en subir les inconvénients et donc de ne plus devoir arbitrer entre personnalisation et protection de la vie privée. C'est au concept de «personnalisation protectrice des données personnelles » - Privacy-enhanced personalization (Kobsa 2007) - ou encore de personalization for privacy (p4p) (Chellappa et Shivendu 2007) que l'on fait référence ici. Ce type de personnalisation ne s'opposerait en effet pas au respect de la vie privée. Elle permettrait même théoriquement une personnalisation plus efficace car respectueuse de l'individu.

\subsection{Limites de l'étude et prolongements possibles}

Cette étude comprend plusieurs limites qui doivent être soulignées mais qui représentent toutefois autant de pistes d'amélioration et de recherches futures.

La première limite concerne la représentativité de l'échantillon interrogé. Comme nous l'avons indiqué, nos répondants sont plus jeunes et plus expérimentés que l'internaute «moyen». Si cet aspect limite en partie le caractère généralisable de nos résultats à l'ensemble de la population francophone (et britannique) utilisant Internet, il leur confère toutefois une valeur prospective puisqu'ils représentent les internautes de demain. Puisqu'une part de plus en plus importante des citoyens Européens accède à Internet d'une part et devient davantage expérimentée d'autre part, leurs attitudes et comportements à l'égard du dévoilement devraient se rapprocher de ceux trouvés ici. De plus, si nous trouvons des différences d'attitudes au sein d'une population jeune et assez homogène, elles devraient se retrouver à l'identique, voire être renforcées sur une population globale plus hétérogène. Nos résultats donnent donc une photo utile - même si elle n'est que partielle - des comportements de dévoilement sur Internet en France et en Angleterre. De plus, le fait que nos résultats soient - au moins en partie - vérifiés sur un échantillon français d'internautes adultes (auteur 2006)

\footnotetext{
${ }^{18}$ Il s'agit pour le site d'adapter son contenu/l'information fournie selon la catégorie à laquelle appartient le visiteur (par exemple : actionnaire, client, fournisseur, demandeur d'emploi, journaliste), sans que ce dernier ait pour autant l'obligation de fournir son identité. Plutôt que l'entreprise affecte d'elle-même le visiteur à une catégorie selon ce qu'elle sait de lui, c'est le visiteur lui-même qui choisit sa catégorie, d'où le terme d'autosegmentation.

${ }_{19} \mathrm{Si}$ ce dispositif rend difficile le rattachement du nom d'emprunt à la véritable identité de la personne, l'anonymat finit souvent par être levé, notamment dans le cas d'un achat en ligne avec livraison physique.
} 
et sur l'échantillon anglais donne une validité certaine à ces quatre groupes. Ceci est d'autant plus vrai que ce découpage en quatre a déjà été trouvé dans les travaux existants (même si les groupes trouvés ici diffèrent de ceux indiqués dans la littérature) qui considèrent majoritairement (e.g. Sheehan 2002) que la typologie originelle en 3 groupes n'est pas réplicable sur les internautes et que la meilleure partition possible sur Internet est de quatre.

Une deuxième limite majeure est liée à la nature déclarative des résultats. Ainsi, nous établissons la typologie sur la base de préférences et de comportements déclarés qui sont parfois différents de ceux réellement adoptés. Là encore, cette étude constitue une première exploration du comportement de dévoilement d'informations personnelles qu'il conviendrait de compléter par une démarche plus réaliste, par exemple à travers des protocoles expérimentaux. Dans cette lignée, certains travaux (auteur) postulent l'existence d'un effet de contexte dans le comportement plus ou moins «bienveillant» des individus confrontés à une sollicitation de leurs données personnelles. Cette idée est d'ailleurs confortée par nos résultats qui confirment, notamment, l'influence de la nature de la relation (i.e. la familiarité) avec le site concerné. Ainsi, il existerait une tendance générale (mais pas automatiquement stable) de l'individu à l'égard de l'échange de données, mise en évidence à travers notre typologie, qui pourrait cependant - dans certains cas - être modérée par l'effet du contexte.

La troisième limite tient à la nature mono-média des résultats. Le questionnaire ne nous permet en effet aucunement de savoir si ces individus se comporte(raie)nt de la même façon sur un support autre qu'Internet, nous empêchant ainsi d'établir toute conclusion concernant la stabilité de ces comportements tous médias confondus. Bien qu'Internet (qu'il soit consulté depuis un ordinateur ou un mobile) constitue aujourd'hui le principal outil de collecte de données, une étude complémentaire pourrait être menée pour comparer les comportements de dévoilement sur différents médias.

Plusieurs pistes peuvent être envisagées pour valider ou compléter nos résultats. En premier lieu, la reconduite de la présente étude sur un échantillon plus représentatif de l'ensemble de la population (et pas seulement des jeunes) constituerait une validation de la typologie obtenue, en même temps qu'une voie de généralisation. Au niveau théorique, il semble encore possible d'approfondir la compréhension de la vie privée en ligne et du comportement d'échange de données personnelles. Il conviendrait notamment de mieux comprendre les motivations liées à ces quatre groupes. Par exemple, qu'est-ce qui explique le comportement de «marchandage» de certains et la «bienveillance» des autres ? De tels comportements sont-ils stables au fil du temps et quelles que soient les circonstances ? Des études comparatives et longitudinales permettraient sans doute de mieux comprendre le phénomène. D'un point de vue empirique, il conviendrait à présent de tester la validité d'un 
cadre conceptuel intégrateur, visant notamment à évaluer l'effet de la préoccupation envers le respect de la vie privée sur la propension à délivrer des données sur le web. L'ajout de facteurs «situationnels », comme le type d'interlocuteur concerné et le niveau de familiarité avec celui-ci ou encore le contexte dans lesquelles les données sont demandées, permettrait d'enrichir la portée, parfois réduite, des recherches actuelles. A cette fin, une approche expérimentale semble particulièrement appropriée, de manière à travailler sur des comportements réels plutôt que déclarés.

\section{Conclusion}

Le travail empirique présenté ici a pour objectif d'établir une typologie des internautes en s'appuyant sur les avantages recherchés lors de la divulgation de données personnelles. L'échantillon de 2014 Français et 1258 Anglais peut être scindé en quatre classes regroupant des internautes aux comportements nettement différenciés.

Bien qu'exploratoire, ce travail est le premier à comparer deux populations issues d'un même Continent. Il souligne que la question du respect de la vie privée en ligne ne peut être envisagée de manière uniforme. Cet aspect est essentiel du point de vue des pratiques car les sites commerciaux, concernés par le recueil des données clients et par la validité de ces données, doivent pouvoir se doter de stratégies adaptatives. La mise en évidence de quatre grands profils d'individus (i.e. les réticents, les désintéressés, les bienveillants et les négociateurs) constitue un premier pas vers une détection plus opérationnelle des comportements de dévoilement de données personnelles. Cette dernière est également facilitée par l'effet potentiellement discriminant de plusieurs types de variables: sociodémographiques (genre, CSP, niveau d'études et expérience d'Internet), attitudinales (confiance dans Internet, préoccupation de vie privée et perception du niveau de protection réglementaire) et comportementales (niveau de protection et de dévoilement). Nos résultats amènent à proposer une personnalisation du e-commerce sans identification dont la généralisation dépend moins de l'émergence d'un modèle économique viable que de l'efficacité des solutions proposées. Les défis techniques et éthiques permettant une meilleure personnalisation et une prise en compte réelle des attentes de vie privée des individus demeurent plus que jamais présents. 


\section{Références}

Arcand M. et Nantel J. (2005), a website's privacy policy: a double-edged sword: risks and benefits when consumers read your privacy statements, cahier de recherche HEC Montréal $\mathrm{n}^{\circ} 05-10-2$

Beauvois J-L. et Joulé R-V. (1981), Soumission et idéologies. Psychosociologie de la rationalisation, Paris, P.U.F.

Belen V. (2005), Les tentatives de protection des données personnelles des individus : difficultés de définition et risques nouveaux, Market Management 2005/2 (Volume 5), 65-80

Belleil A. (2007), Personnalisation sans identification, Internet Actu, www.internetactu.net/2007/10/22/personnalisation-sans-identification/

Brandtzæg, P.B. (2010), Towards a unified Media-User Typology (MUT): A meta-analysis and review of the research literature on media-user typologies. Computers in Human Behavior, 26(5), 940-956.

Burnett G. (2000), Information exchange in virtual communities: a typology, Information Research, 5, 4, July 2000, http://informationr.net/ir/5-4/paper82.html

Cassel L. et Wolz U. (2001), Client side personalization, Proceedings of the Second DELOS Network of Excellence Workshop on Personalisation and Recommender Systems in Digital Libraries No. 01/W03, Dublin City University, Ireland, www.ercim.org/publication/wsproceedings/DelNoe02/CasselWolz.pdf

Castaneda A. J and Montoro F. J (2007), The effect of Internet general privacy concern on customer behaviour, Electronic Commerce Research, 7, 117-141

Chellappa R. K and Shivendu S. (2007), An economic model of privacy: a property rights approach to regulatory choices for online personalization, Journal of Management Information Systems, 24, 3, 193-225

Cranor L., Reagle J. et Ackerman M. (1999), Beyond concern: understanding net users' attitudes about online privacy, AT\&T Labs, Rapport de Recherche, 4, 3.

Culnan, M.J. et Armstrong, P.K. (1999), Information Privacy Concerns, Procedural Fairness, and Impersonal Trust: An Empirical Investigation. Organization Science, 10(1), 104-115.

Dinev T. et Hart P. (2005-06), Internet privacy concerns and social awareness as determinants of intention to transact, International Journal of Electronic Commerce, 10, 2 (Winter), 7-29.

Dinev T. (2006), Bellotto M., Hart P., Russo V., Serra I. and Colautti C. (2006) Privacy calculus model in e-commerce, a study of Italy and the United States, European Journal of Information Systems, 15, 389-402

Ding, C. \& He, X. (2004), K-means clustering via principal component analysis. In Proceedings of the twenty-first international conference on Machine learning. Banff, Alberta, Canada: ACM, p. 29. Available at: http://portal.acm.org/citation.cfm?id=1015330.1015408 [Accessed July 16, 2010].

Farag N. et Krishnan M. (2003), An empirical evaluation of information features and the willingness to be profiled online for personalization, http://misrc.umn.edu/workshops/2003/spring/farag_030703.pdf

Freestone, O. \& Mitchell, V. (2004), Generation Y Attitudes Towards E-ethics and Internetrelated Misbehaviours. Journal of Business Ethics, 54(2), 121-128

Freytag, P.V., et Clarke, A.H. (2001), Business to business market segmentation, Industrial Marketing Management, 30, 473- 486. 
Fukuyama F (1995), Trust: the Social Virtues and the Creation of Prosperity, Free Press, New York.

Hann I. et alii (2002), On line information privacy: measuring the cost-benefit trade-off, 23td International Conference on Information Systems, http://www.comp.nus.edu.sg/ ipng/research/privacy_icis.pdf

Hofstede, G. (2001), Cultural consequences: Comparing values, behaviors, institutions, and organizations across nations ( $2^{\text {nd }} E d$.). Thousand Oaks, CA: Sage.

Hough, M. (2009), Keeping it to ourselves: Technology, privacy, and the loss of reserve. Technology in Society, 31(4), 406-413.

Hui Kai-Lung, Teo Hock Hai, Sang-Yong Tom Lee. (2007), The value of privacy assurance; an exploratory field experiment, MIS Quarterly, 31(1), 19-33.

Hunt, S. D. (1983). Marketing theory: The philosophy of marketing science. Homewood, IL: Richard D. Irwing, Inc.

Isaac H. et Kalika (2001), Organisation, technologies de l'information et vie privée, Revue Française de Gestion, 134, 101-105

Jensen C., Potts C. et Jensen C. (2005), Privacy practices of Internet users: self-reports versus observed behaviour, International Journal of Human Computer Studies, Special issue on HCI Research in Privacy and Security, Octobre, 63(1-2), 203-227.

John et Srivastava (1999), The Big Five trait taxonomy: History, measurement, and theoretical perspectives. In L. A. Pervin \& O. P. John (Eds.), Handbook of personality: Theory and research (2nd ed., pp. 102-138). New York: Guilford.

Johnson, G. \& Kulpa, A. (2007), Dimensions of online behaviour: Toward a user typology. Cyberpsychology and Behavior, 10(6), 773-779.

Kiesler C.A. (1971), The psychology of commitment. Experiments linking behaviour to belief, New York, Academic Press.

Kimery, K.M. et M. McCord (2002), Third-party Assurances: The Road to Trust in Online Retailing, Proceedings of the $35^{\text {th }}$ Annual Hawaii International Conference on Systems Sciences.

Kimmel A. (1996), Ethical issues in behavioural research: A study, Cambridge, MA: Blackwell

Kobsa A. (2007), Privacy-enhanced personalization, Communications of the ACM, 50, 8, 2433

LaPlaca, P.J. (1997), Contributions to marketing theory and practice from industrial marketing management, Journal of Business Research, 38, 179- 198

Laufer R. et Wolfe M. (1977), Privacy as a concept and a social issue: a multidimensional developmental theory, Journal of Social Issues, 33, 22-42

Lemoine P. (1993), Le commerce dans la société informatisée, Economica.

Lemoine P. (2000), Commerce électronique, marketing et liberté, Travaux du groupe d'études société de l'information et vie privée, Académie des Sciences Morales et Politiques, Rapport final (2), Chapitre 7, http://www.asmp.fr

Liu C., Marchewkab J. T., Luc J., Yu C-S (2005), Beyond concern-a privacy-trustbehavioral intention model of electronic commerce, Information and Management, 42, 289304 
Lueg, J.E., Ponder N., Beatty S.E., and Capella M.L. (2006), Teenagers' use of alternative shopping channels: a consumer socialization perspective. Journal of Retailing 82, 2, p137153.

Lusoli W. et Miltgen C. (2009), Young people and emerging digital services: an exploratory survey on motivations, perceptions and acceptance of risks, JRC Scientific and Technical Reports EUR 23765 EN, W. Lusoli, R. Compañó \& I. Maghiros (Eds.), Sevilla: EC JRC IPTS, http://ftp.jrc.es/EURdoc/JRC50089.pdf

Malhotra, N., Kim, S. \& Agarwal, J. (2004), Internet users' information privacy concerns (IUIPC): The construct, the scale, and a causal model. Information Systems Research, 15(4), 336-355.

Mascarenhas, O.A.J., Kesavan, R. \& Bernacchi, M.D. (2003), Managing online privacy: a call for joint ownership. Journal of Consumer Marketing, 20(7), 686 - 702.

McKnight, D.H., Choudhury, V. \& Kacmar, C. (2002), Developing and Validating Trust Measures for e-Commerce: An Integrative Typology. Info. Sys. Research, 13(3), 334-359.

McLean D. (1995) Privacy and its invasion. Wesport, CT: Praegar

Milberg S., Burke S., Smith J. et Kallman E. (1995), Values, personal information, privacy and regulatory approaches, Communication of the ACM, 38, 12, 65-74

Ortega Egea J.M., Recio Menéndez M. and Román González M.V. (2007), Diffusion and usage patterns of Internet services in the European Union, Information Research 12 (2), p. 302.

Pavlou P. (2003), Consumer acceptance of electronic commerce: integrating trust and risk with the technology acceptance model, International Journal of Electronic Commerce, 7, 3, 69-103

Raghu, T.S.; Kannan, P.K.; Rao, H.R.; et Whinston, A.B. (2001), Dynamic profiling of consumers for customized offerings over the Internet: A model and analysis, Decision Support Systems, 32, 2, 117-134.

Ray, Michael (1982), Advertising and Communication Management, Englewood Cliffs, NJ: Prentice Hall, Inc

Rennhard M. et al. (2004), Towards Pseudonymous e-Commerce, Electronic Commerce Research, 4, 1, 83-111.

Rogers E.M (2003), Diffusion of Innovations ( $5^{\text {th }}$ ed.) New York: Free Press

Román, S. et Cuestas, P.J. (2008), The Perceptions of Consumers Regarding Online Retailers' Ethics and Their Relationship with Consumers' General Internet Expertise and Word of Mouth: A Preliminary Analysis. Journal of Business Ethics, 83(4), 641-656.

Roman, S. (2006), The Ethics of Online Retailing: A Scale Development and Validation from the Consumers' Perspective. Journal of Business Ethics, 72(2), 131-148.

Schoeman F. (1984), Privacy: Philosophical Dimensions of the Literature, dans Philosophical Dimensions of Privacy: An Anthology, Schoeman ed, New York: Cambridge University Press

Schwartz B. (2000), Charting a privacy research agenda: responses, agreements, and reflections, Connecticut Law Review, 929

Sheehan K. (2002), Toward a typology of internet users and online privacy concerns, The Information Society, 18, 21-32. 
Spiekermann S., Grossklags J. et Berendt B. (2001), E-privacy in $2^{\text {nd }}$ generation e-commerce: privacy preferences versus actual behaviour, ACM Conference on Electronic Commerce. Tampa, 14-17 Octobre, 38-47.

Taylor D., Altman I. et Sorrentino R. (1969), Interpersonal exchange as a function of rewards and costs and situational factors : expectancy confirmation-disconfirmation, Journal of Experimental Social Psychology, 5, 324-339

Terré F. (2000), Vie privée, Travaux du groupe d'études société de l'information et vie privée, Académie des Sciences Morales et Politiques, Rapport final (3), Chapitre 7, http://www.asmp.fr

Thiétart et al. (2003), Méthodes de recherche en management, Dunod, Paris

Tweney, D. (1998), Lack of Trust Hurts Consumer Commerce for Online Retailers, Infoworld, (20:19), May 11, p. 77.

Van Slyke, C. et al. (2006), Concern for information privacy and online consumer purchasing. Journal of the Association for Information Systems, 7(6), 415-444.

Ward S., Bridges K. et Chitty B. (2005), Do incentive matter? An examination of online privacy concerns and willingness to provide personal and financial information, Journal of Marketing Communications, 11, 1, 21-40

Wedel, M., et Kamakura. W A. (2000), Market Segmentation: Conceptual and Methodological Foundations. Boston, MA: Kluwer.

Westin A. (1967), Privacy and Freedom, NY: Atheneum Press.

Westin A. et Harris \& Associates (1996), Equifax-Harris consumer privacy survey, Atlanta, GA: Equifax Inc.

Whitley, E.A. (2009). Informational privacy, consent and the "control" of personal data. Information Security Technical Report, 14(3), 154-159.

Woodman R. et al. (1982), A survey of employee perceptions of information privacy in organizations, Academy of Management Journal, 25, 3, 647-663

Zillien, N. \& Hargittai, E. (2009), Digital Distinction: Status-Specific Types of Internet Usage. Social Science Quarterly, 90(2), 274-291. 
Annexe 1 - Synthèse des principales typologies de consommateurs dans la littérature sur la préoccupation pour le respect de la vie privée

\begin{tabular}{|c|c|c|c|c|}
\hline $\begin{array}{l}\text { Auteur } \\
\text { (année) }\end{array}$ & $\begin{array}{c}\text { Population étudiée } \\
\text { (taille de l'échantillon) }\end{array}$ & $\begin{array}{l}\text { Base de la typologie } \\
\text { (variables analysées) }\end{array}$ & Types de consommateurs trouvés & Variables discriminantes \\
\hline Westin (1996) & Consommateurs Etasuniens & $\begin{array}{l}3 \text { questions générales sur } \\
\text { l'attitude face au RVP }\end{array}$ & $\begin{array}{l}3 \text { segments trouvés : } \\
\text { - les “ fondamentalistes" }(25 \%) \\
\text { - les “pragmatiques" }(55 \%) \\
\text { - les " non préoccupés" }(20 \%)\end{array}$ & $\begin{array}{l}\text { - sexe : femmes plus préoccupées que les hommes } \\
\text { et ce quel que soit le domaine (collecte, utilisation } \\
\text { secondaire des données, accès, ...) }\end{array}$ \\
\hline $\begin{array}{l}\text { Cranor, } \\
\text { Reagle et } \\
\text { Ackerman } \\
(1999)\end{array}$ & \begin{tabular}{|l|} 
Internautes panélistes \\
Etasuniens \\
405 questionnaires ( 381 \\
conservés pour l'analyse)
\end{tabular} & $\begin{array}{l}\text { Questions sur l'attitude générale } \\
\text { face au RVP et intentions de } \\
\text { réponse à des sollicitations de } \\
\text { données personnelles (dans } \\
\text { différentes conditions) }\end{array}$ & $\begin{array}{l}3 \text { segments trouvés : } \\
\text { - les “ fondamentalistes" }(17 \%) \\
\text { - les "pragmatiques" }(56 \%) \\
\text { - les “peu préoccupés" }(27 \%)\end{array}$ & $\begin{array}{l}\text { - aucune différence sociodémographique } \\
\text { - sensibilité perçue des données similaire dans } \\
\text { tous les segments } \\
\text { - les “fondamentalistes" sont les plus opposés au } \\
\text { transfert automatique de leurs données }\end{array}$ \\
\hline $\begin{array}{l}\text { Spiekermann } \\
\text { Grossklags et } \\
\text { Berendt } \\
(2001)\end{array}$ & $\begin{array}{l}\text { Membres d'une université } \\
\text { allemande (95\% étudiants) } \\
206 \text { questionnaires (171 } \\
\text { conservés pour l'analyse) }\end{array}$ & $\begin{array}{l}\text { Questions sur la préoccupation } \\
\text { pour le RVP (protection des } \\
\text { données personnelles) }\end{array}$ & $\begin{array}{l}\text { segments trouvés : } \\
\text { - " peu préoccupés " }(24 \%) \\
\text { - " préoccupés par leur identité " }(20 \%) \\
\text { - " préoccupés par leur profil " }(26 \%) \\
\text { - " fondamentalistes " }(30 \%)\end{array}$ & $\begin{array}{l}\text { - les "peu préoccupés " sont ceux qui refusent le } \\
\text { moins de se dévoiler } \\
\text { - continuité de la communication (flow) : les } \\
\text { "préoccupés par leur identité " ont un " flow" } \\
\text { faible, ceux " préoccupés par leur profil "élevé }\end{array}$ \\
\hline $\begin{array}{l}\text { Sheehan } \\
\text { (2002) }\end{array}$ & $\begin{array}{l}\text { Internautes Etasuniens } \\
3724 \text { questionnaires ( } 889 \\
\text { complétés) } \\
\text { échantillon représentatif des } \\
\text { internautes Etasuniens }\end{array}$ & $\begin{array}{l}\text { Niveau de préoccupation face à } \\
15 \text { situations de collecte de } \\
\text { données personnelles }\end{array}$ & $\begin{array}{l}4 \text { segments trouvés : } \\
\text { - " alarmés " }(3 \%) \\
\text { - " prudents" }(43 \%) \\
\text { - " circonspects" }(38 \%) \\
\text { - " non préoccupés" }(16 \%)\end{array}$ & $\begin{array}{l}\text { - sexe et niveau de revenu non discriminants } \\
\text { - âge : non préoccupés et alarmés plus âgés } \\
\text { - niveau d'éducation : non préoccupés et } \\
\text { circonspects sont moins “ éduqués" } \\
\text { - utilisation d'Internet non discriminante } \\
\text { - comportements de divulgation discriminants : } \\
\text { l'enregistrement sur des sites, la fourniture } \\
\text { d'informations incomplètes ou erronées }\end{array}$ \\
\hline $\begin{array}{l}\text { Hann, Hui, } \\
\text { Lee et Png } \\
\text { (2002) }\end{array}$ & $\begin{array}{l}\text { Etudiants Etasuniens (84) et } \\
\text { de Singapour (184) } \\
268 \text { questionnaires (243 } \\
\text { classés dont } 78 \text { Etasuniens) }\end{array}$ & $\begin{array}{l}\text { Questions sur les bénéfices } \\
\text { acceptés en échange des données } \\
\text { et sur les types de préoccupations } \\
\text { envers la protection des données }\end{array}$ & $\begin{array}{l}\text { segments trouvés : } \\
\text { - les " gardiens de la VP" }(72 \% \text { pour US et } \\
83 \% \text { pour Singapour }) \\
\text { - les "vendeurs de données" }(20 \% \text { et } 8,5 \%) \\
\text { - les " chercheurs de confort" }(8 \% \text { et } 8,5 \%)\end{array}$ & $\begin{array}{l}\text { - dans l'échantillon US, les “vendeurs de } \\
\text { données " ont une plus grande expérience de } \\
\text { dévoilement de données personnelles }\end{array}$ \\
\hline $\begin{array}{l}\text { Jensen Potts } \\
\text { et Jensen } \\
(2005)\end{array}$ & $\begin{array}{l}\text { Internautes volontaires } \\
\text { (175 majoritairement } \\
\text { Etasuniens dont } 93 \\
\text { conservés) }\end{array}$ & $\begin{array}{l}5 \text { questions reflétant l'attitude } \\
\text { face au RVP }\end{array}$ & $\begin{array}{l}3 \text { segments trouvés : } \\
\text { - les " fondamentalistes" }(34 \%) \\
\text { - les "pragmatiques" }(43 \%) \\
\text { - les " non préoccupés" }(23 \%)\end{array}$ & $\begin{array}{l}\text { - sexe : les femmes sont plus préoccupées que les } \\
\text { hommes pour les } 5 \text { questions } \\
\text { - expérience d'achat en ligne non discriminante } \\
\text { - pas d'effet sur la probabilité de lire une charte }\end{array}$ \\
\hline
\end{tabular}


Annexe 2 - Instrument de collecte

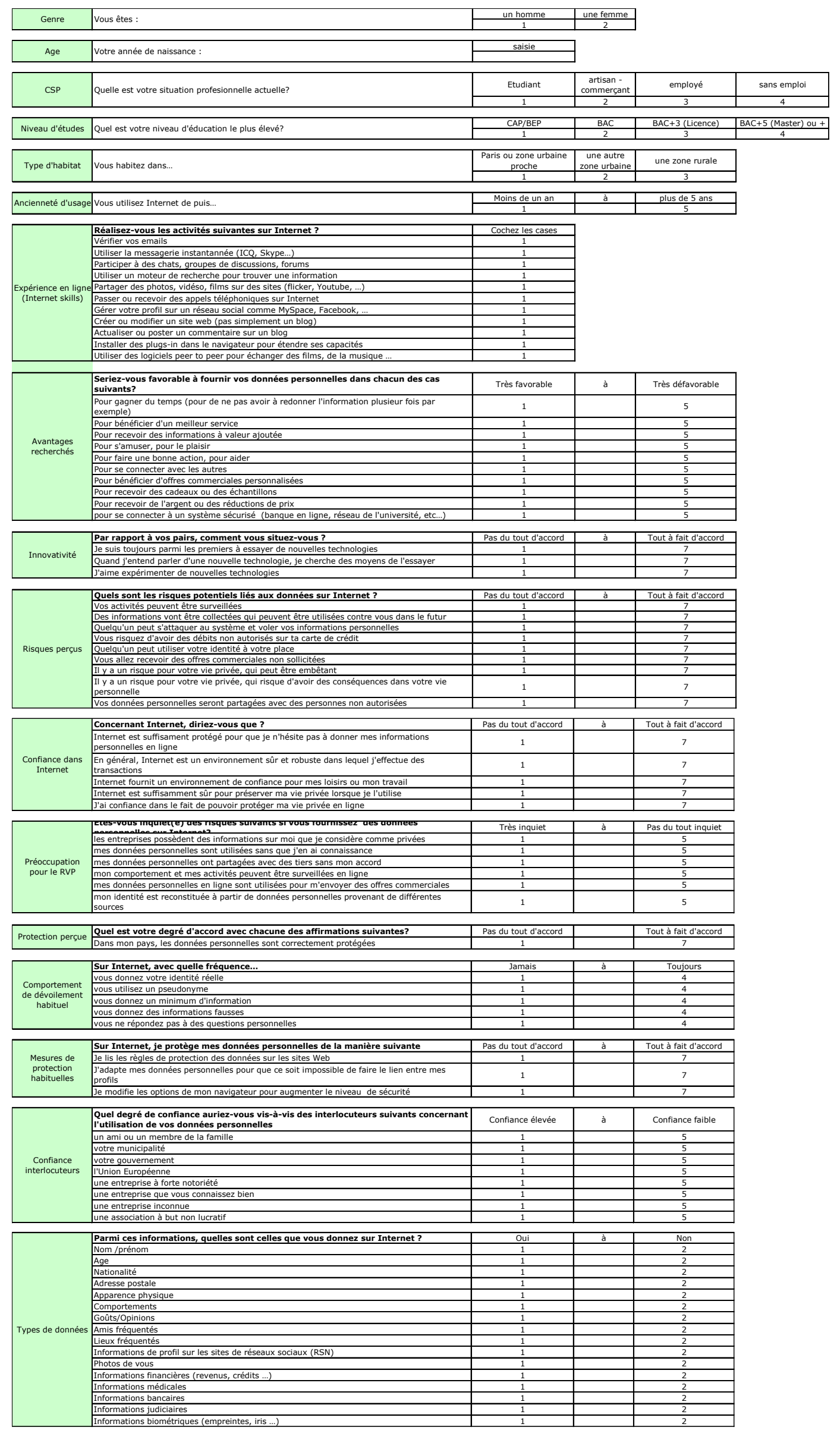


Annexe 3 - Comparaison des caractéristiques de l'échantillon et de la population (au niveau français et anglais)

\begin{tabular}{|c|c|c|c|c|c|}
\hline & & $\begin{array}{l}\text { Population des jeunes internautes } \\
\text { français (12-24 ans) } \\
\text { (Forrester sept. 2007) }\end{array}$ & $\begin{array}{c}\text { Echantillon de l'enquête } \\
\text { 15-25 ans, France } \\
\text { (mi 2008) }\end{array}$ & $\begin{array}{c}\text { Population des jeunes } \\
\text { internautes anglais (16-24 ans) } \\
\text { (National Statistics UK 2008) }\end{array}$ & $\begin{array}{c}\text { Echantillon de l'enquête } \\
\text { 15-25 ans, GB } \\
\text { (mi 2008) }\end{array}$ \\
\hline $\begin{array}{l}\text { Usage du } \\
\text { web }\end{array}$ & $\begin{array}{l}\text { Connexion à Internet } \\
\text { chaque jour }\end{array}$ & $82 \%$ & $96 \%$ & $93 \%$ & $94 \%$ \\
\hline \multirow{6}{*}{$\begin{array}{l}\text { Activités } \\
\text { régulières } \\
\text { en ligne }\end{array}$} & $\begin{array}{l}\text { Participation à un } \\
\text { chat/forum/newsgroup }\end{array}$ & $43 \%$ & $38 \%$ & $37 \%$ (16-19 ans) & $30 \%$ \\
\hline & Envoi d'emails & $89 \%$ & $99 \%$ & $91 \%$ & $98 \%$ \\
\hline & $\begin{array}{l}\text { Utilisation de moteurs de } \\
\text { recherche }\end{array}$ & $69 \%$ & $96 \%$ & $77 \%$ & $93 \%$ \\
\hline & $\begin{array}{l}\text { Publication ou maintien } \\
\text { d'un blog }\end{array}$ & $37 \%$ & $37 \%$ & $18 \%$ (16-19 ans) & $8 \%$ \\
\hline & $\begin{array}{l}\text { Utilisation de sites de } \\
\text { réseaux sociaux }\end{array}$ & $24 \%$ & $50 \%$ & $55 \%(2007)$ & $54 \%$ \\
\hline & $\begin{array}{l}\text { Utilisation de la } \\
\text { messagerie instantanée }\end{array}$ & $75 \%$ & $86 \%$ & ND (Non Disponible) & $65 \%$ \\
\hline & & $\begin{array}{l}\text { Population des internautes français } \\
\text { (Médiamétrie, juin 2006) }\end{array}$ & $\begin{array}{c}\text { Echantillon de l'enquête } \\
\text { 15-25 ans, France } \\
\text { (mi 2008) }\end{array}$ & $\begin{array}{c}\text { Population des jeunes } \\
\text { internautes anglais } \\
\text { (source) }\end{array}$ & $\begin{array}{c}\text { Echantillon de l'enquête } \\
\text { 15-25 ans, GB } \\
\text { (mi 2008) }\end{array}$ \\
\hline Genre & $\begin{array}{l}\text { Masculin } \\
\text { Féminin }\end{array}$ & $\begin{array}{l}51 \% \\
49 \%\end{array}$ & $\begin{array}{l}40 \% \\
60 \%\end{array}$ & ND & $\begin{array}{l}65 \% \\
35 \%\end{array}$ \\
\hline Age & $\begin{array}{l}15-18 \text { ans } \\
19-21 \text { ans } \\
22-25 \text { ans }\end{array}$ & $\begin{array}{l}36 \% \\
28 \% \\
36 \%\end{array}$ & $\begin{array}{l}10 \% \\
31 \% \\
59 \%\end{array}$ & ND & $\begin{array}{l}41 \% \\
29 \% \\
30 \%\end{array}$ \\
\hline Profession & $\begin{array}{l}\text { Salariés } \\
\text { Sans activité dont } \\
\text { Scolaires/étudiants } \\
\text { Sans emploi }\end{array}$ & $\begin{array}{l}32 \% \\
61 \% \\
7 \%\end{array}$ & $\begin{array}{l}34 \% \\
65 \% \\
9 \%\end{array}$ & $\begin{array}{l}52 \% \\
38 \% \\
9 \%\end{array}$ & $\begin{array}{l}53 \% \\
38 \% \\
9 \%\end{array}$ \\
\hline
\end{tabular}


Annexe 4 - Répartition en 5 groupes dans les deux pays

\section{Graphique de cinq groupes dans l'échantillon français}

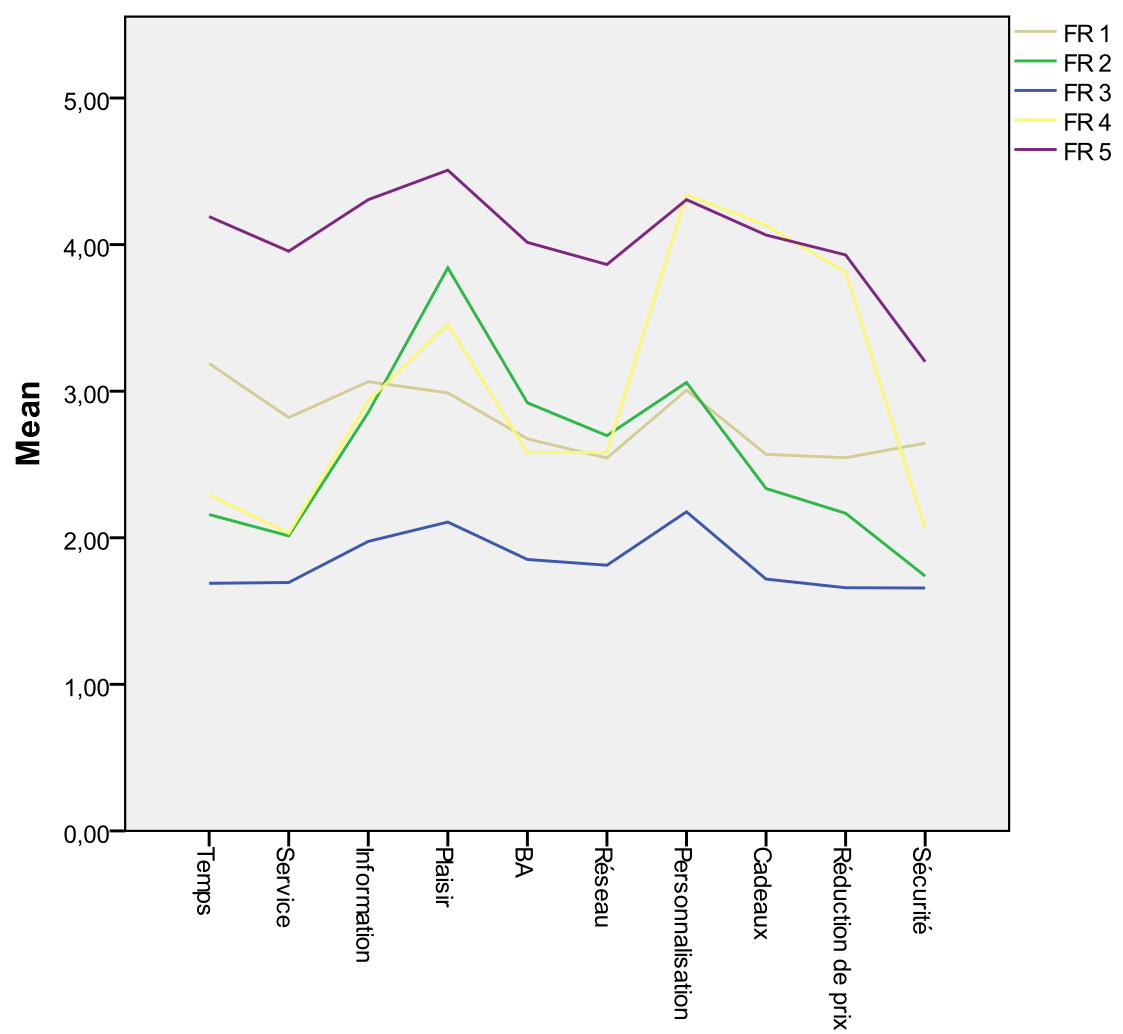

Graphique de cinq groupes dans l'échantillon anglais

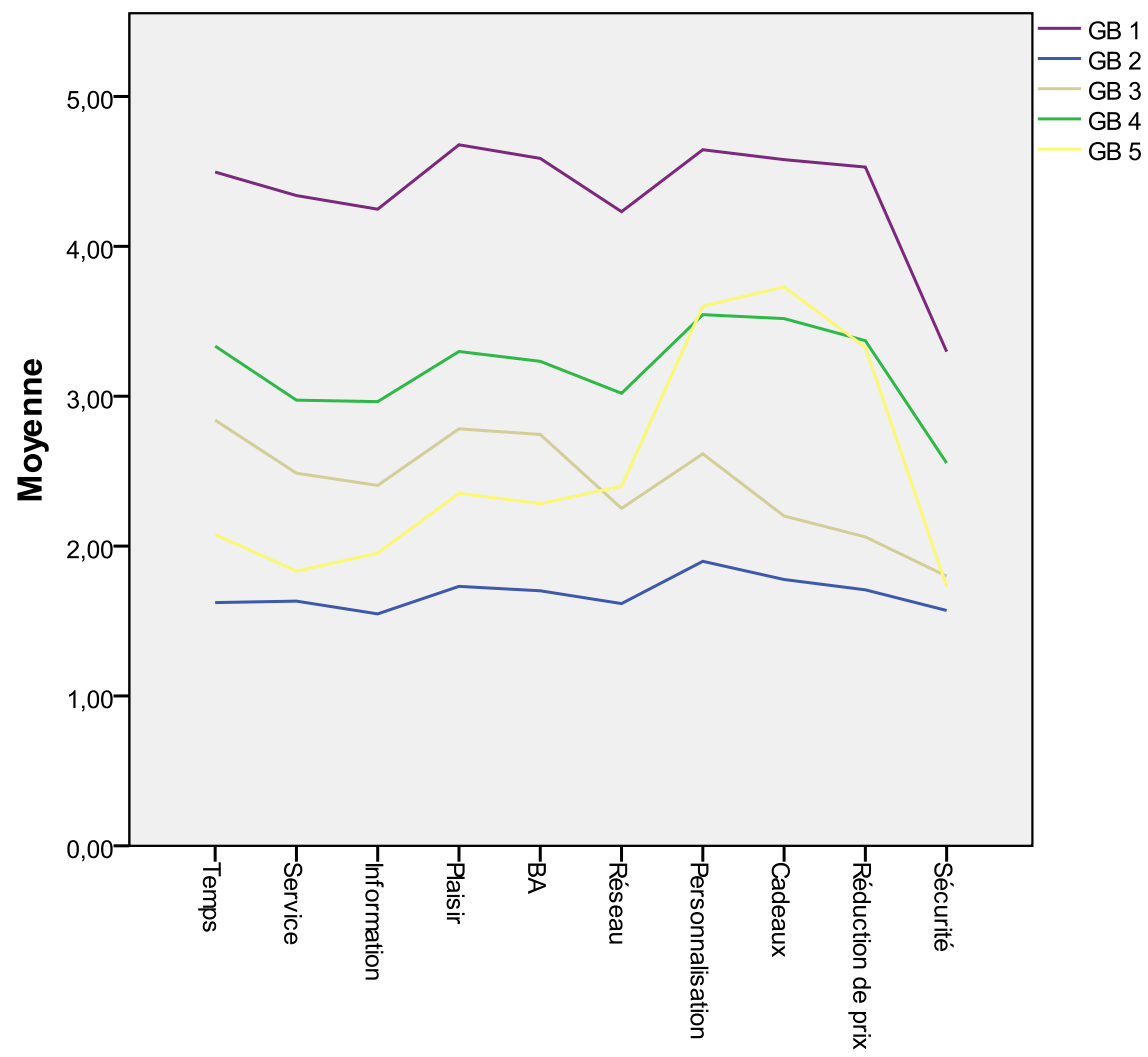


Annexe 5 - Classement des avantages recherchés dans chaque classe selon le pays ${ }^{20}$

\section{Classement des avantages recherchés dans l'échantillon français}

\begin{tabular}{|c|c|c|c|c|c|c|c|}
\hline Classes / Avantages & $\begin{array}{c}1 \\
\text { Bienveillants } \\
(\mathrm{N}=648)\end{array}$ & $\begin{array}{c}2 \\
\text { Désintéressés } \\
(\mathrm{N}=434)\end{array}$ & $\begin{array}{c}3 \\
\text { Négociateurs } \\
(\mathrm{N}=695)\end{array}$ & $\begin{array}{c}4 \\
\text { Réticents } \\
(\mathrm{N}=237)\end{array}$ & $\left|\begin{array}{c}\text { TOTAL } \\
(\mathrm{N}=2014)\end{array}\right|$ & $\mathrm{F}$ & Sig. \\
\hline Avantage temps & 1,73 & 2,13 & 2,86 & 4,02 & 2,48 & 518,7 &, 000 \\
\hline Avantage service & 1,73 & 1,94 & 2,56 & 3,69 & 2,29 & 469,8 &, 000 \\
\hline Avantage information & 2,07 & 2,71 & 3,08 & 4,26 & 2,81 & 498,8 &, 000 \\
\hline Avantage plaisir & 2,23 & 3,28 & 3,55 & 4,45 & 3,17 & 466,6 &, 000 \\
\hline $\begin{array}{l}\text { Avantage rendre } \\
\text { service / BA }\end{array}$ & 1,94 & 2,55 & 2,89 & 3,80 & 2,62 & 311,8 &, 000 \\
\hline $\begin{array}{l}\text { Avantage connexion } \\
\text { réseau }\end{array}$ & 1,85 & 2,49 & 2,74 & 3,70 & 2,51 & 342,8 &, 000 \\
\hline $\begin{array}{l}\text { Avantage } \\
\text { personnalisation }\end{array}$ & 2,23 & 4,09 & 3,01 & 4,42 & 3,16 & 629,9 & ,000 \\
\hline Avantage cadeaux & 1,76 & 3,83 & 2,39 & 4,20 & 2,71 & 1183,7 &, 000 \\
\hline $\begin{array}{l}\text { Avantage réduction de } \\
\text { prix }\end{array}$ & 1,70 & 3,49 & 2,33 & 4,05 & 2,58 & 860,5 &, 000 \\
\hline Avantage sécurité & 1,69 & 2,02 & 2,25 & 3,06 & 2,11 & 138,4 &, 000 \\
\hline
\end{tabular}

\section{Classement des avantages recherchés dans l'échantillon anglais}

\begin{tabular}{l|cccc|c|c|c}
\hline Classes / Avantages & $\begin{array}{c}1 \\
\text { Bienveillants } \\
(\mathrm{N}=469)\end{array}$ & $\begin{array}{c}\text { Désintéressés } \\
(\mathrm{N}=250)\end{array}$ & $\begin{array}{c}\text { Négociateurs } \\
(\mathrm{N}=389)\end{array}$ & $\begin{array}{c}\text { Réticents } \\
(\mathrm{N}=150)\end{array}$ & $\begin{array}{c}\text { TOTAL } \\
(\mathrm{N}=1258)\end{array}$ & $\mathrm{F}$ & Sig. \\
\hline Avantage temps & 1,84 & $\mathbf{2 , 5 6}$ & 3,12 & 4,39 & 2,68 & 347,4 &, 000 \\
Avantage service & 1,76 & $\mathbf{2 , 0 8}$ & $\mathbf{2 , 8 9}$ & 4,13 & 2,45 & 509,3 &, 000 \\
Avantage information & 1,67 & 2,14 & 2,87 & 4,11 & 2,43 & 583,4 &, 000 \\
Avantage plaisir & 1,92 & 2,75 & 3,07 & $\mathbf{4 , 5 5}$ & 2,76 & 538,1 &, 000 \\
$\begin{array}{l}\text { Avantage rendre } \\
\text { service / BA }\end{array}$ & $\mathbf{1 , 9 0}$ & 2,58 & 3,08 & 4,43 & 2,70 & 507,2 &, 000 \\
$\begin{array}{l}\text { Avantage connexion } \\
\text { réseau }\end{array}$ & 1,78 & 2,76 & 2,62 & 4,02 & 2,50 & 273,5 &, 000 \\
$\begin{array}{l}\text { Avantage } \\
\text { personnalisation }\end{array}$ & 2,15 & $\mathbf{3 , 9 9}$ & 2,93 & $\mathbf{4 , 5 7}$ & 3,04 & 513,2 &, 000 \\
Avantage cadeaux & 1,97 & $\mathbf{4 , 0 7}$ & $\mathbf{2 , 7 4}$ & 4,50 & 2,93 & 719,8 &, 000 \\
$\begin{array}{l}\text { Avantage réduction de } \\
\text { prix }\end{array}$ & $\mathbf{1 , 8 7}$ & $\mathbf{3 , 6 6}$ & $\mathbf{2 , 6 2}$ & 4,43 & 2,76 & 547,4 &, 000 \\
Avantage sécurité & $\mathbf{1 , 6 0}$ & 1,88 & 2,31 & 3,13 & 2,06 & 92,6 &, 000 \\
\hline
\end{tabular}

${ }^{20}$ Les notes sur lesquels les individus sont les plus réservés (notes élevées) sont indiquées en gris foncé pour les anglais et en noir dans le cas des français. 